\title{
Identification and characterization of $B r x R$ as a regulatory gene in the BREX phage restriction system
}

Yvette Luyten ${ }^{1}$, Deanna Hausman², Juliana Young², Lindsey A. Doyle², Natalia C. UbillaRodriquez ${ }^{2}$, Abigail R. Lambert ${ }^{2}$, Corina Arroyo², Kevin Forsberg,"\#, Richard M. Morgan ${ }^{1}$, Barry L. Stoddard ${ }^{2 \star}$ and Brett K. Kaiser ${ }^{3,{ }^{*}}$

\author{
${ }^{1}$ New England Biolabs \\ 240 County Road \\ Ipswich, MA 01938, USA
}

2 Division of Basic Sciences

Fred Hutchinson Cancer Research Center 1100 Fairview Ave. North, Seattle WA 98109 USA

${ }^{3}$ Department of Biology Seattle University 901 12th Avenue Seattle, WA 98122, USA

\# Current address:

Microbiology Department UT Southwestern Medical Center 6000 Harry Hines Blvd, Dallas TX 75390 USA

*Co-corresponding authors (kaiserb@seattleu.edu; bstoddar@fredhutch.org ) 
Bacteriophage exclusion ('BREX') phage restriction systems are found in a wide range of bacteria. Various BREX systems encode unique combinations of proteins that usually include a site-specific methyltransferase; none appear to contain a nuclease. Here we describe the identification and characterization of a Type I BREX system from Acinetobacter and the effect of deleting each BREX ORF on growth, methylation and phage restriction. The analysis identified a previously uncharacterized gene at the 5' end of the BREX operon that is dispensable for methylation but involved in restriction. Biochemical and crystallographic analyses of this factor, which we term BrxR ('BREX Regulator'), demonstrate that it forms a homodimer and specifically binds a pseudo-palindromic DNA target site upstream of its transcription start site. Precise deletion of the BrxR gene causes cell toxicity, reduces phage restriction, and significantly increases the expression of BrxC. In contrast, the introduction of a premature stop codon into the BrxR gene has little effect, implying that the BrxR coding sequence and BrxR protein have independent functional roles in BREX regulation. We speculate that the BrxR coding sequence is involved in cis regulation of BREX activity and that the BrxR protein may play an additional regulatory role, perhaps during horizontal transfer of the system. 
Bacteria and phage are locked in an ancient conflict that has driven bacteria to evolve numerous and varied anti-phage defense systems, including restriction-modification systems (RM), CRISPR-Cas, abortive infection mechanisms (abi) and toxin-antitoxin (TA) gene pairs (1-7). These defense systems operate either by discriminating self-genomes from non-self and degrading foreign DNA (RM and CRISPR-Cas) or by inducing dormancy or a cell suicide response (abi and TA systems) (2).

BREX is a largely uncharacterized defense system that restricts phage by a poorly understood mechanism $(8,9)$. First described in S. coelicolor in the 1980 s as being associated with a "phage growth limited" (pgl) phenotype (10), the BREX family was subsequently found to comprise at least 6 subfamilies that are widely distributed in bacteria and archaea (present in $\sim 10 \%$ of genomes) $(2,8)$. The various subtypes typically contain four to eight genes drawn from at least 13 gene families. DNA modification is a central feature of BREX function; five of six subfamilies encode a site-specific DNA methyltransferase (PgIX), while the sixth family encodes a PAPS reductase gene that also modifies host DNA (8). The most abundant family (BREX type 1) is present in $\sim 55 \%$ of BREX-containing genomes and encodes several large ORFs (>75 kDa) with identifiable enzymatic motifs, including an ATPase domain (BrxC), an alkaline phosphatase domain (PglZ), and a AAA+ ATPase (BrxL) with homology to Lon protease and/or RadA. How these factors collaborate in a BREX restriction system is not understood.

Two Type 1 BREX systems (from E. coli and B. cereus) and one Type 2 BREX system (from S. coelicolor; previously referred to as 'phage growth limited' or 'Pgl') have been described in detail. The timing and mechanism of phage restriction appears to differ between these systems. Bacteria harboring Type 2 BREX systems are sensitive to an initial round of phage infection (and permit lysogeny) but are then resistant to phage emerging from that cycle $(10,11)$. In contrast, type 1 systems appear to halt phage production prior to the first round of infection at a step very early after phage absorption, and also prevent lysogenization $(8,9)$. Both systems utilize PgIX-directed DNA methylation to distinguish self from non-self, but do not appear to restrict phage by degrading their genome, and no BREX ORFs appear to encode a nuclease $(8,9)$. BREX also does not appear to function by an abortive infection (abi) or cell suicide (TA) mechanism (8). Thus, the mechanism of BREX-mediated restriction appears to be novel from previously described defense systems.

BREX systems may employ various strategies to regulate or suppress inherently toxic activities during horizontal transfer and/or after establishment in an existing bacterial host until needed. Such fail-safe mechanisms have been described for other phage restriction systems. They include transcriptional repressors such as $\mathrm{C}$ proteins in R-M systems (which delay expression of a nuclease upon transfer to a new host) $(12,13)$, direct inhibitors of restriction factors such as the TRIP13 factor in CBASS restriction systems (which attenuate activity until detection of phage) (14), and phase variation mechanisms (15). In Type 2 BREX systems, PgIX and PgIZ appear to form a toxin/anti-toxin pair (16), and additional interactions between BREX factors have been proposed to regulate toxic activities (9). The Type 1 BREX 
system from B. cereus contains two operons (BrxA, BrxB, BrxC and PlgX on one; PglZ and BrxL on the other), further suggesting that BREX systems might be appropriately organized for transcriptional regulation (8). Finally, phase variation of PgIX has been observed in the type II BREX system from Streptomyces coelicolor (16-18); a high frequency of disruptions in PgIX coding sequences may indicate that phase variation may be a frequent mechanism of BREX regulation (8).

Two recent studies of host-virus dynamics in Vibrio and Proteus bacterial species, performed in India and Ireland, respectively, have further illustrated the global distribution and likely role of a broad family of related transcriptional regulators in various phage restriction systems including $\operatorname{BREX}(19,20)$. Clinical isolates of Vibrio cholera contain a $\sim 100 \mathrm{~kb}$ mobile genetic element (the SXT integrative conjugative element) that encodes various defense systems (including BREX) within a genetic hotspot (19). Analysis of neighboring genes in 76 distinct isolates revealed that an ORF encoding a WYL domain is present upstream of the defense systems (19).

WYL proteins have previously been linked to numerous CRISPR/Cas genetic loci (21) and shown to transcriptionally regulate CRISPR transcripts in several cyanobacterial species (22). WYL proteins also transcriptionally regulate numerous cellular pathways, including DNA damage response $(23,24)$. WYL domains are frequently encoded on the same polypeptide with two other domains-a DNA binding winged helix-turn-helix ( $w H T H)$ domain and a WCX domain-- and have been proposed to function as sensors that bind to environmental ligands -- perhaps nucleic acids or second messengers -- that allosterically activate the associated DNA binding domain on the same polypeptide (21-24).

In this study we characterize a bacterial Type 1 BREX system from Acinetobacter. We identify its PgIX methyltransferase target site, determine the requirements of BREX ORFs for PgIX-directed methylation, and then further study the requirements of the same ORFs for phage restriction. We identify a 5' ORF often linked to Type I BREX systems and show that this gene-which we call BrxR-is required for phage restriction but dispensable for methylation. We demonstrate that $B r x R$ is a sequence-specific DNA binding protein comprising $\mathrm{HTH}, \mathrm{WYL}$ and WCX domains, propose that the protein and its reading frame act respectively as trans and cis transcriptional regulators of the BREX system, and suggest that its function has been widely adopted in more divergent bacterial species to regulate defense systems.

\section{METHODS}

Identification and subcloning of a novel type I BREX system. Whole genome sequencing of Acinetobacter sp. NEB394 was carried out using Pacific Biosciences (PacBio) SMRT sequencing technology (25). The assembled genome and associated plasmids have been submitted to NCBI (NCBI:txid2743575). Putative DNA modification motifs were identified using PacBio SMRTAnalysis 2.3.0 Modification_and_Motif_Analysis_1.0. To assign methylation function to DNA methyltransferase genes, 
putative DNA methyltransferase genes identified within REBASE (26) and flanking genes were examined using the standard protein blast (BLASTP) program from NCBI (27). One putative N6A DNA methyltransferase appeared as part of a classic BREX system with flanking genes closely related to the BrxA, BrxB, BrxC, PglZ, and BrxL genes (8).

Oligonucleotide primers used in these studies (Supplementary Table S1) were synthesized by Integrated DNA Technology (Coralville, IA). Molecular biology reagents including Q5 Hot Start HighFidelity DNA polymerase, NEBuilder HiFi DNA assembly master mix, restriction enzymes, DNA size standards, and competent cells were provided by New England Biolabs. Genomic DNA isolation, plasmid purification, agarose gel and PCR clean ups were performed using Monarch DNA kits (NEB). Plasmid DNA constructs were confirmed by sequencing on the ABI 3130xI capillary machine (Applied Biosystems) and PacBio RSII (Pacific Biosciences).

The 14.1kb BREX operon was PCR amplified as three $\sim 4.7 \mathrm{~Kb}$ fragments from genomic DNA using overlapping primers designed for NEBuilder HiFi assembly. The expression plasmid pACYC184 (28) was amplified by inverse PCR to insert the BREX operon downstream of the Tet promoter. The Acinetobacter sp. NEB394 BREX system was initially subcloned in the pACYC184 expression vector under the control of a constitutive Tet promoter, using NEBuilder HiFi DNA assembly mix. Briefly, PCR-amplified insert and plasmid DNAs were mixed in equimolar ratios, incubated at $50^{\circ} \mathrm{C}$ for 60 minutes, and transformed into ER2683 competent cells per manufacturer's instructions. Individual colonies were selected and grown overnight in LB broth (10 g/L soy peptone, $5 \mathrm{~g} / \mathrm{L}$ yeast extract, $5 \mathrm{~g} / \mathrm{L} \mathrm{NaCl}, 1 \mathrm{~g} / \mathrm{L} \mathrm{MgCl}, 1 \mathrm{~g} / \mathrm{L}$ dextrose) supplemented with chloramphenicol $(25 \mu \mathrm{g} / \mathrm{ml})$. Total DNA was isolated from overnight cultures and prepared for sequencing on PacBio RSII (Pacific BioSciences, Menlo Park, CA).

In a second round of subcloning to further investigate possible regulatory interactions between the BrxR protein and a putative cis regulatory sequence upstream of the Acinetobacter BREX transcript, the constitutive tet promoter was replaced by the insertion of a 97 basepair sequence that immediately precedes the BREX operon. The introduction of that sequence was carried out in two sequential rounds of PCR. First round amplifications were performed using Q5 Hot Start High-Fidelity DNA polymerase (NEB) and primer pairs brxRBD_for1/brxRBD_rev1 (wild-type BREX, BrxR-Stop, and BrxR-R47A) or brxRBD_delR_for1/brxRBD_rev1 (BrxR gene deletion) (Supplementary Table S1) under the following conditions: 25 cycles at $\mathrm{T}_{a}=66^{\circ} \mathrm{C}$ and 9-minute extension. Primer pairs were designed to introduce 70 of 93 basepair sequence into the wild-type and point mutation BREX constructs and 61 of 93 basepair sequence into the BrxR gene deletion construct in the first round of amplification. Following amplification, template DNA was removed by digestion with Dpnl for 30 minutes at $37^{\circ} \mathrm{C}$. PCR products were analyzed by agarose gel electrophoresis and purified using Monarch PCR purification kit. Purified products were used as template in the second round of amplification. Primer pairs, brxRBD_for2/brxRBD_rev2 and brxRBD_deIR_for2/brxRBD_deIR_rev2 were designed to insert the remainder of the desired sequence 
upstream of the BREX operon (23 and 32 basepairs, respectively) and to contain 24 and 32 base overlaps for Gibson assembly post-amplification. Amplification was performed using Q5 Hot Start HighFidelity DNA polymerase (NEB) under the same cycling parameters described for round 1 amplification. Following amplification, the desired $\sim 17 \mathrm{~Kb}$ band was gel purified using Monarch Gel Purification kit and purified amplicons were self-ligated using NEBuilder HiFi DNA Assembly Master Mix and transformed into ER2683 competent cells.

Individual colonies were grown overnight in LB broth supplemented with chloramphenicol (25 $\mu \mathrm{g} / \mathrm{ml})$. Plasmid DNAs were isolated from overnight cultures for analysis by restriction digestion and PacBio Sequel II sequencing of the entire plasmid. The size of plasmid constructs was determined by digestion with Hpal in a $30 \mu \mathrm{l}$ reaction containing $5 \mathrm{ng}$ template DNA, 1x NEB CutSmart Buffer, and $1 \mu \mathrm{l}$ restriction enzyme, at $37^{\circ} \mathrm{C}$ for 30 minutes. Digestion products were analyzed by agarose gel electrophoresis. Insertion of the 93 basepair sequence was verified via Sanger sequencing (ABI 3130xI capillary machine) and the entire construct sequence ( $17 \mathrm{~kb})$ was confirmed via sequencing on the PacBio Sequel II system (Pacific Biosciences).

Pacific Biosciences Sequencing. In vivo modification activities and sequence specificity of the wildtype BREX construct and subsequent gene deletion or point mutation plasmids were analyzed by sequencing on the PacBio RSII (Pacific Biosciences). Determination of in vivo DNA modifications and corresponding target motifs was performed using PacBio SMRTAnalysis 2.3.0 Modification_and_Motif_Analysis_1.0. Prior to library preparation, input DNA was sheared to an average size of 5-10 kb using gTubes (Covaris) and concentrated using 0.6V Ampure beads (Pacific Biosciences). Libraries were prepared according to manufacturer's protocol using the SMRT-bell Template Prep kit 1.0 (Pacific Biosciences) for sequencing on the PacBio RSII (Pacific Biosciences).

Transcription start-site (TSS) analysis. Three independent cultures of Acinetobacter sp. NEB394 were grown at $30^{\circ} \mathrm{C}$ in $\mathrm{LB}$ media to late log phase $\left(\mathrm{OD}_{600}\right.$ between $\left.0.7-0.75\right)$. Two volumes RNA protect (Qiagen) was added to the culture prior to RNA extraction using Monarch total RNA miniprep kit (NEB). Isolated RNA was quantified using Quibit RNA BR assay kit (Invitrogen) and used for Cappable-seq and SMRT-Cappable-seq.

Using the Cappable-seq method (29), two biological replicate enriched transcriptional start-site (TSS) libraries were generated. Briefly, a capping reaction was performed using vaccinia capping enzyme (NEB) to add a desthio-biotinylated GTP (DTB-GTP) cap to the 5' triphosphate terminus of RNA. The capped RNA was fragmented using T4 polynucleotide kinase and primary RNA transcript isolated through 2 rounds of streptavidin magnetic bead enrichment. Following enrichment, the DTB-GTP cap was removed leaving a 5' monophosphate terminus using RNA 5'pyrophophohydrolase (NEB), RNA was bound to AMPure beads and eluted in low TE (10mM Tris pH8.0, 0.1mM EDTA pH8.0). Eluted RNA was used for library preparation using the NEBNext small RNA library kit (NEB). The enriched and control 
libraries were amplified through 16 cycles and 10 cycles of PCR, respectively. RNA sequencing was performed on Illumina MiSeq ${ }^{\circledR}$ with single reads of 100 bases using V3 Illumina platform.

Single-end Illumina reads were trimmed to remove adaptors using cutadapt (30) with default parameters. Reads were mapped to the Aceinetobacter sp NEB 394 plasmid pBspH3 (CP055285.1) using Bowtie2 v2.3.4.3(31). Gene annotations were derived from the NCBI Aceinetobacter sp 394 plasmid pBspH3 annotation (GenBank: CP055285.1). Mapped reads were visualized using Integrative Genomics Viewer (32).

Total RNA from a third biological replicate was used to prepare a SMRT-cappable-seq library with minor modifications to the previously described method (33). Second-strand cDNA was bulk amplified with LongAmp HotStart Taq using CapSeq_for_dU_RM1 and CapSeq_rev_dU_RM1 primers. cDNA was size selected using Sage BluePippin (Sage Science) at a cut-off threshold of 2.5kb. Size-selected cDNA was PCR amplified, purified and PacBio SMRTbell adapters were ligated as previously described. Non-sizeselected and size-selected SMRTbell libraries were sequenced using the PacBio RSII and Sequel platforms.

Gene Deletions and point mutations. Individual genes were systematically removed from the cloned BREX operon (pAcBREX_WT) by inverse PCR. The pAcBREX_WT was amplified using Q5 Hot Start High-Fidelity DNA polymerase (NEB) and primers containing a 15-21 base overlap (see primer table) targeting DNA sequences immediately adjacent to the 5' and 3' ends of the gene to be removed.

Following amplification, template DNA was removed by digestion with Dpnl for 30 minutes at $37^{\circ} \mathrm{C}$. PCR products were analyzed by agarose gel electrophoresis and purified using Monarch gel extraction or PCR purification kits. Purified amplicons were self-ligated using NEBuilder HiFi DNA Assembly Master Mix and transformed into ER2683 competent cells to generate a series of 7 plasmid constructs each

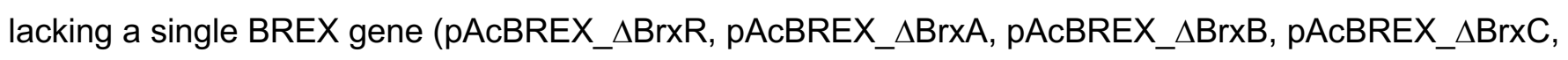

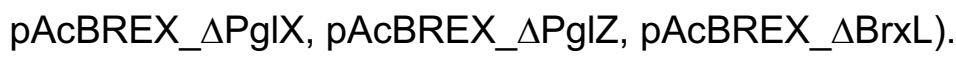

Individual colonies were grown overnight in LB broth supplemented with chloramphenicol (25 $\mu \mathrm{g} / \mathrm{ml})$. Plasmid and total DNAs were isolated from overnight cultures for analysis by restriction digestion and PacBioRSII sequencing respectively. Restriction enzymes selected for screening contained 2 recognition sites within the PAcBREX_WT plasmid, 1 within and 1 outside of the gene to be removed. Plasmid

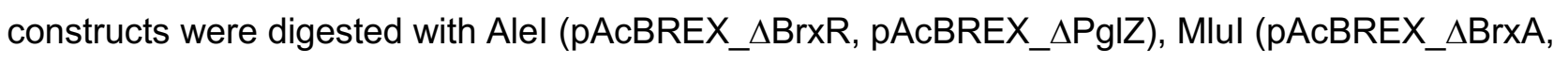

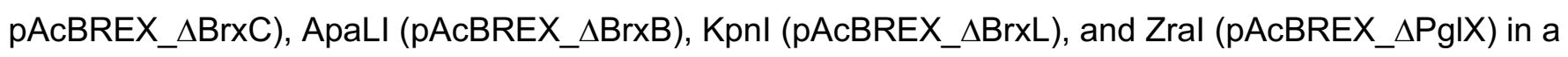
$30 \mu \mathrm{l}$ reaction containing $0.5 \mathrm{ng}$ template DNA, $1 \mathrm{x}$ NEB CutSmart Buffer, and $1 \mu \mathrm{l}$ restriction enzyme, at $37^{\circ} \mathrm{C}$ for 30 minutes. Digestion products were analyzed by agarose gel electrophoresis. The ligation junction for plasmids producing the predicted restriction patterns were confirmed via Sanger sequencing (ABI 3130xl capillary machine). Upon confirmation by restriction digestion and Sanger sequencing, total 
DNA derived from the corresponding overnight culture was used to generate PacBio libraries analyzed for in vivo modification activity and sequence confirmation of the expression plasmid.

Bacterial growth and phage restriction assays. All infections using phage $\lambda$ were performed with a virulent mutant of the phage unable to undergo lysogeny $\left(\lambda_{\text {vir }}\right)(34)$. In all experiments, cells were transformed with pACYC-based plasmids encoding the wild-type BREX operon, various deletion mutants, or control vectors that lack the BREX operon. Transformants were stored at $-80^{\circ} \mathrm{C}$ as glycerol stocks. Experiments were performed both in E. coli NEB 5-alpha cells (New England Biolabs) and in E. coli strain ER2683 (New England Biolabs).

All experiments began using isolated colonies derived from freezer stocks, which were then used to inoculate $4 \mathrm{ml}$ of lysogeny broth (LB; $10 \mathrm{~g} / \mathrm{L}$ casein peptone, $10 \mathrm{~g} / \mathrm{L} \mathrm{NaCl}, 5 \mathrm{~g} / \mathrm{L}$ ultra-filtered yeast powder) supplemented with $1.25 \mathrm{mM} \mathrm{MgCl}_{2}, 1.25 \mathrm{mM} \mathrm{CaCl}_{2}$, and $25 \mathrm{mg} / \mathrm{ml}$ chloramphenicol ('overnight culture media'). Cultures were grown overnight at $37^{\circ} \mathrm{C}$ with shaking at $220 \mathrm{rpm}$. The next day, overnight cultures were diluted 50 -fold in $4 \mathrm{ml}$ of LB supplemented with $1.25 \mathrm{mM} \mathrm{MgCl}, 1.25 \mathrm{mM} \mathrm{CaCl}_{2}, 0.2 \%$ maltose, and $25 \mu \mathrm{g} / \mathrm{ml}$ chloramphenicol ('outgrowth media') and grown to mid-log (absorbance readings at $600 \mathrm{~nm}$ varied between 0.2 and 0.6 ) at $37^{\circ} \mathrm{C}$, shaking at $220 \mathrm{rpm}$. Mid-log cultures were then diluted to an $\mathrm{OD}_{600}$ of 0.01 in LB containing $1.25 \mathrm{mM} \mathrm{MgCl}_{2}, 1.25 \mathrm{mM} \mathrm{CaCl}_{2}, 25 \mathrm{mg} / \mathrm{ml}$ chloramphenicol. Phage titers were determined via a plaque assay to ensure accurate MOls. Phage $\lambda_{\text {vir }}$ samples were serially diluted in $\mathrm{SM}$ buffer (50 mM Tris- $\mathrm{HCl}, 25 \mathrm{mM} \mathrm{NaCl}, 4 \mathrm{mM} \mathrm{MgSO}_{4}$ ) before use and added to the bacterial samples at multiplicities of infection (MOls) ranging from 0.001 to 1.0. SM buffer alone was added to samples without phage. Cultures were then arrayed in triplicate across a 96-well plate (Greiner cat\#655083, $100 \mu \mathrm{l}$ per well) and grown for ten hours in a BioTek Cytation three plate reader at $37^{\circ} \mathrm{C}$ with continuous orbital shaking at 282 cycles per minute. Absorbance readings were taken every 15 minutes at $600 \mathrm{~nm}$.

Additional, complementary phage plaque formation assays were performed using the same titred phage stock and overnight cultures of ER2683 grown in overnight culture media. Multiple dilutions of the overnight cultures were generated the next morning (between 30- and 100-fold) in outgrowth media and then grown to an $\mathrm{OD}_{600}$ of approximately 0.4 to 0.5 . 80 microliters of each sample were mixed with $3 \mathrm{~mL}$ of top agar (0.5\% agar in LB supplemented with $1.25 \mathrm{mM} \mathrm{MgCl}_{2}, 1.25 \mathrm{mM} \mathrm{CaCl}_{2}$, and $25 \mu \mathrm{g} / \mathrm{ml}$ chloramphenicol) and applied to bottom agar plates (1.5\% agar in LB supplemented with $1.25 \mathrm{mM}$ $\mathrm{MgCl}_{2}, 1.25 \mathrm{mM} \mathrm{CaCl}_{2}$, and $25 \mu \mathrm{g} / \mathrm{ml}$ chloramphenicol). Plates were allowed to solidify and dry for approximately 15 minutes and then spotted with $5 \mu$ of 10 -fold serial dilutions (ranging from $10^{1}$ to $10^{8}$ dilutions) of titred phage. Plates were incubated overnight at $37^{\circ} \mathrm{C}$ and examined the following morning for plaque formation. Each experiment was performed with a minimum of three biological replicates.

Protein expression and purification. The BrxR gene was subcloned from the native Acinetobacter 394 locus into the pET15b plasmid to contain an N-terminal, thrombin-cleavable 6XHistidine (His6) tag. 
Inductions were carried out in BL21 (DE3) pLysS E. coli cells. For inductions, a 10-mL overnight starter culture was grown in LB media with ampicillin $(100 \mu \mathrm{g} / \mathrm{mL})$, diluted 100 -fold into the same media and incubated at $37^{\circ} \mathrm{C}$ with shaking until an $\mathrm{OD}_{600}$ of 0.6 was reached. IPTG was added to a final concentration of $500 \mu \mathrm{M}$, and the culture was shaken for an additional 18-22 hours at $16^{\circ} \mathrm{C}$. Cell pellets were harvested by centrifugation and stored at $-20^{\circ} \mathrm{C}$.

Cell pellets were resuspended in lysis buffer (25 mM Tris (pH 7.5), $300 \mathrm{mM} \mathrm{NaCl}, 20 \mathrm{mM}$ imidazole)), lysed by sonication on ice and centrifuged in an SS34 rotor for 25 minutes at 18,000 rpm. The supernatant was filtered through a $5 \mu \mathrm{m}$ syringe filter, and the clarified lysate was incubated in batch with Ni-NTA agarose resin (Qiagen) for 1 hour at $4^{\circ} \mathrm{C}$ with rotation. The resin was transferred to a gravity filtration column (Bio-Rad), washed with $>50$ volumes of lysis buffer at $4^{\circ} \mathrm{C}$ and eluted in lysis buffer supplemented with $200 \mathrm{mM}$ imidazole. Biotinylated thrombin (EMD Millipore) was added to the eluted protein (1 unit of thrombin per mg of protein), and the sample was dialyzed into $200 \mathrm{mM} \mathrm{NaCl}, 50 \mathrm{mM}$ Tris $(\mathrm{pH} 7.5)$ overnight at $4^{\circ} \mathrm{C}$. Removal of the His6 tag was assessed by running "pre-thrombin" and "post-thrombin" samples on a 4-12\% BOLT SDS PAGE gel in MES buffer (Invitrogen); the difference in size between the His6-tagged and untagged version was clearly resolved on these gels. The sample was concentrated to $2 \mathrm{~mL}$ in an Amicon Ultra centrifugal filter (10,000 molecular weight cutoff; Millipore). Thrombin was removed by incubation with streptavidin agarose (Novagen) for 30 minutes at $4^{\circ} \mathrm{C}$. The sample was filtered through a $0.22 \mu$ m centrifugal filter and loaded onto a HiLoad 16/60 Superdex 200 prep grade size exclusion column (Millipore Sigma) equilibrated in $25 \mathrm{mM}$ Tris (pH 7.5), $200 \mathrm{mM} \mathrm{NaCl}$. Peak fractions were pooled and concentrated to $14 \mathrm{mg} / \mathrm{mL}$. Single-use aliquots were flash frozen in liquid nitrogen and stored long-term at $-80^{\circ} \mathrm{C}$. This untagged BrxR (i.e with the His 6 tag removed) was used in biochemical and crystallography experiments.

To produce selenomethionyl (SeMet)-containing BrxR for crystallization, we followed a previously described induction and growth protocol (35) using the same plasmid, E. coli strain and purification strategy described above. This protein was concentrated and stored at $12 \mathrm{mg} / \mathrm{mL}$.

DNA binding assays. DNA substrates for gel shift assays were amplified by PCR using Q5 Polymerase (New England Biolabs) and purified using a DNA Clean and Concentrator kit (Zymo Research). DNA was used at a final concentration of $20 \mathrm{nM}$ in binding experiments. Untagged BrxR proteins were diluted in $100 \mathrm{mM} \mathrm{NaCl}, 25 \mathrm{mM}$ Tris (pH 7.5) and used at final concentrations ranging from $25 \mathrm{nM}$ to $800 \mathrm{nM}$. Binding reactions were assembled in binding buffer (20 mM Tris ( $\mathrm{pH} 7.9), 40 \mathrm{mM} \mathrm{NaCl}, 2.5 \%$ sucrose)) and incubated at $22^{\circ} \mathrm{C}$ for 30 minutes. Samples were resolved on native acylamide gels containing $0.5 \mathrm{X}$ TBE buffer and $7 \%$ acrylamide (made from a stock of $40 \%$ acrylamide with a ratio of $29: 1$ acrylamide:bisacrylamide; Bio-Rad). The running buffer for the gels was 1 XTBE. Prior to loading samples, the gels were pre-run for 30 minutes at 90 volts in 1 X TBE buffer and the wells were flushed using a syringe. $4 \mu$ of each binding reaction was loaded on the gel (without dye) and run at room 
temperature for 100 minutes at 90 volts. Gels were stained for 30 minutes in SybrGold (Invitrogen) diluted in 1X TBE and visualized on a Typhoon scanner using a $488 \mathrm{~nm}$ laser and Cy2 filter.

BrxR crystallographic structure determination. The structure of apo BrxR was initially determined to $2.3 \AA$ resolution via single anomalous dispersion (SAD) phasing using SeMet-derivatized protein. The crystals were grown by hanging drop vapor diffusion in drops set with $1 \mu$ l of protein (12 mg/mL) plus $1 \mu \mathrm{l}$ of well solution (10\% PEG3000, 0.1 M HEPES (pH 7.3), 2\% benzamidine-HCL). For cryopreservation, crystals were transferred into crystallization solution containing $20 \%$ ethylene glycol and $0.1 \% \mathrm{H}_{2} \mathrm{O}_{2}$, incubated for one minute and flash frozen in liquid nitrogen. Diffraction data for the SeMet-containing BrxR was collected at the Advanced Light Source synchrotron facility (ALS, Berkeley, CA) at beamline 5.0.2. A single-wavelength data set was collected with an incident $x$-ray wavelength of $0.9792 \AA$, corresponding to the anomalous dispersion peak for selenium (12.662 keV).

Data were indexed and scaled using HKL2000 software (36). Experimental phases were determined by SAD phasing in CC4Pi's CRANK phasing and building pipeline (37), producing an initial model with refinement Rwork and Rfree values of 0.24 and 0.28 , respectively. Subsequent rounds of building were carried out in Coot (38) and refinement was performed using Refmac5 (39).

To visualize the DNA-bound form of BrxR, ssDNA oligonucleotides containing BrxR's putative binding site (ATACCGTAAAAATAATTTACTGTAT top strand; bottom strand is complementary) were synthesized, annealed to produce blunt-end double strand DNA (dsDNA) and purified by HPLC (Integrated DNA Technologies). $150 \mu \mathrm{M}$ dsDNA was incubated with $125 \mu \mathrm{M}$ BrxR and crystallized by hanging drop diffusion in $0.04 \mathrm{M}$ Citric acid, 20\% w/v PEG 3350, 60 mM BIS-TRIS propane (pH 6.4). Crystals were cryopreserved in the same solution containing $22.2 \%$ ethylene glycol prior to freezing in liquid nitrogen. A native dataset was collected on ALS Beamline 5.01. The structure of the DNA-BrxR complex was determined by molecular replacement at $2.3 \AA$ resolution using PHENIX (40), with the previously determined structure of the unbound protein as a molecular probe and phasing model. One copy of the protein homodimer was identified in the crystallographic asymmetric unit, with LLG and TFZ scores of 423.6 and 24.7 , respectively. Density corresponding to the bound DNA target site was immediately identifiable in electron density difference maps, and the corresponding bound DNA was then manually fit using COOT (38). The structure of the DNA-bound BrxR complex was refined using PHENIX (40).

\section{RESULTS}

Identification and characterization of a novel type I BREX system and its PgIX methyltransferase. We initiated this project to better understand the role of DNA methylation in Acinetobacter with the intent of identifying all methylated sites in a representative genome as well as the DNA methyltransferases 
(MTases) responsible for those modifications. For this purpose, we chose bacterial strain NEB 394 (Acinetobacter 394), which was known to encode several R-M systems and carried out PacBio genome sequencing and corresponding methylome analysis of Acinetobacter 394 genomic DNA. Identified among the observed uniformly methylated sites identified was a non-palindromic sequence corresponding to $5^{\prime}-\mathrm{GTAG}\left(\mathrm{A}^{\mathrm{m} 6}\right) \mathrm{T}-3^{\prime}$, with methylation observed at the adenine base in the fifth position of the motif. Motif Software called $99.85 \%$ of the sites in the genome as methylated (1318 of 1320 sites) at 180X coverage, indicating essentially complete modification.

Next, we compiled a list of DNA MTases present in the Acinetobacter 394 genome (chromosome and plasmids), based on sequence similarity to known DNA MTases identified using the REBASE bioinformatic pipeline (26), that might target this motif. One plausible candidate was a gamma-class amino DNA methyltransferase, members of which have been shown to hemi-methylate sites at nonpalindromic positions. To test the activity of this candidate, we subcloned the MTase gene and introduced it into $E$. coli under control of a constitutive promoter and then analyzed the methylome of the E. coli chromosome by PacBio sequencing. However, we observed no novel methylation events at the same sequence motif (or others) by this analysis.

We therefore examined the genomic locus surrounding the candidate MTase, to identify additional neighboring genes that might be required for the methylation activity. This analysis revealed that the Acinetobacter 394 MTase was flanked by genes known to be conserved across type I BREX phage restriction systems, indicating that it is a BREX-associated PgIX enzyme. The BREX system from Acinetobacter 394 contained the same conserved six genes present in all Type I systems, as well as a seventh gene located immediately upstream of the BREX locus (Figure 1a); we eventually termed this gene BrxR ('BREX regulator'; see below).

To determine if this BREX gene cluster (including PgIX) was sufficient to methylate the target motif, we cloned all seven genes from Acinetobacter genomic DNA into the pACYC184 vector downstream of a constitutive tet promoter and introduced the plasmid into E. coli strain ER2683. PacBio sequencing and methylome analysis of the resulting transformed E. coli indicated full methylation at the GTAGAT motif previously observed in the native Acinetobacter 394 host strain, with the Motif software calling $100 \%$ (1024 of 1024) of the GTAGATD sites in the E. coli genome as methylated at 116X coverage (Figure 1b). PglX-mediated methylation has been reported for three bacterial species ( $E$. coli, $B$. cereus and $L$. casei) $(8,9,41)$; all recognize 6 basepair, non-palindromic motifs and methylate adenine in the fifth position of those targets, as we observe with Acinetobacter $394 \mathrm{PgIX}$.

Four additional BREX genes are required for host methylation in E. coli by PgIX. The observation that Acinetobacter $394 \mathrm{PgIX}$ was not functional when expressed by itself in $E$. coli suggested that it requires other proteins in the BREX system to form a functional DNA MTase. To investigate further, we generated seven additional $\mathrm{pACYC}$ vectors, each harboring the complete BREX system with one 
individual gene precisely deleted, transformed them into E. coli and performed additional PacBio sequencing and methylome analysis on the host E. coli genomic DNA (Figure 1c). Deletion BrxR or BrxL (the last ORF in the BREX gene cluster) did not affect methylation at the target motif. In contrast, deletion of any one of the individual BrxA, BrxB, BrxC or PglZ genes resulted in loss target methylation, implying that these proteins are all required, along with PgIX, to form a functional DNA MTase. These findings are intriguing, as PgIX by itself appears to have all the required domains for methyltransferase function and in most RM systems would be expected to be fully functional. The requirements of other BREX genes for PglX-mediated methylation has only been reported for $E$. coli (9). In that system, which comprises six genes (and does not include BrxR), all BREX components except BrxA and BrxL are required for methylation. The disparity in the requirement of BrxA between Acinetobacter 394 and $E$. coli may reflect differences in mechanism or regulation between the two systems. In all BREX systems it appears that host-protective methylation requires a multi-protein complex consisting of BREX factors.

Deletion of BrxR, BrxB or PgIZ within the BREX operon delays or impairs cell growth. Further growth analyses of $E$. coli transformed with the same pACYC constructs described above (intact BREX or BREX harboring individual gene deletions) indicated that deletion of BrxR, BrxB and PglZ resulted in slower cell growth and/or an extended lag phase before approaching saturation (Figure 1d). The difference between those three BREX gene deletions was also visible when examining the size distribution of individual colonies arising on transformation plates (Supplementary Figure S1). Toxicity caused by deletion of PgIZ has also been reported in the type II BREX system from S. coelicolor, in which PglZ is proposed to function as PglZ as an antitoxin partner to PgIX (16).

\section{The BREX system restricts a lytic phage in E. coli when preceded either by a constitutive tet} promoter or by the native upstream genomic sequence from Acinetobacter. An analysis of the BREX system in Acinetobacter 394 indicated that it was transcribed as a single mRNA, with its transcription start site (TSS) located 23 basepairs upstream of the BrxR gene (Figure 2a and Supplementary Figure S2). We therefore examined the ability of the BREX operon to restrict phage infection and lysis in E. coli when preceded either by a constitutive tet promoter or instead by the $97 \mathrm{bp}$ sequence corresponding to the upstream Acinetobacter 394 genomic region immediately preceding BrxR (which included a predicted bacterial promoter immediately upstream of the Acinetobacter BREX TSS). For these experiments we conducted both plaque formation assays and bacterial growth and lysis assays in liquid culture, and employed a virulent mutant of $\lambda$ phage, termed ' $\lambda_{\text {vir }}$ ', which is unable to undergo lysogeny (34) against E. coli strain ER2683 (an MM294 derivative lacking all known methylation-active restriction systems (42)). The results indicated that in either genetic configuration, the BREX operon restricted phage infection and lysis by approximately equivalent amounts (Figure $\mathbf{2 b , c}$ ).

To further examine the generalizability and reproducibility of these results, we tested the ability of the BREX operon to restrict phage infection and lysis in a different E. coli strain (NEB-5 $\alpha$; New England 
Biolabs). BREX again demonstrated a strong protective effect out to ten hours post-challenge, while cells lacking BREX displayed significant lysis and crash of the culture within five hours of the challenge (Supplementary Figure S3). The level of protection conferred by the intact BREX system displayed a significant dependence on the (MOI) during the initial challenge; cells containing BREX eventually crashed at the highest MOI (0.1) but maintained continued growth at lower MOl's of 0.01 and 0.001 .

All seven BREX subunits, including BrxR, are required for restriction of a lytic phage. We next went on to examine the effect of individually deleting each of the seven BREX genes from the operon in strain ER2683, using both plaque formation assays (Figure 3a) and liquid culture bacterial growth and lysis assays (Figure 3b and Supplementary Figure S4). Unlike the effect of each of the same deletions on host methylation (for which BrxR and BrxL were dispensable) deletion of each of the seven BREX genes led to a significant reduction in phage restriction, with deletion of each impairing phage restriction in liquid culture at both high and low phage MOls (1.0 and 0.01).

The effect of single gene deletions on phage restriction has also been reported for strain $E$. coli $\mathrm{HS}$. In that system, all BREX genes except BrxA were required for phage restriction (9). Individual BREX gene requirements from other systems have not yet been reported.

\section{BrxR is a homodimeric winged helix-turn-helix-WYL-WCX protein with site-specific DNA binding}

function. We were intrigued by the observation that BrxR was required for BREX-mediated phage restriction, since it has not been reported as a core component of Type I BREX systems and is not part of the Type I systems so far described from $E$. coli or $B$. cereus $(8,9)$. Bioinformatic analyses indicated that BrxR contains three domains: an N-terminal winged helix-turn-helix (wHTH) domain, a WYL domain and a 'WYL C-terminal extension' ('WCX') domain. Proteins with this domain architecture are widely present in bacterial genomes and are thought in most cases to function as transcriptional regulators (24). The only structurally characterized HTH-WYL-WCX homolog, PafBC, is a transcriptional regulator of DNA damage responses in mycobacteria $(24,43)$. PafBC is notably different from the majority of $\mathrm{HTH}-$ WYL-WCX proteins, including BrxR homologs, in that it contains a tandem duplication of HTH-WYL-WCX domains on the same polypeptide (proteins of this class represent only $\sim 5 \%$ of all HTH-WYL-WCX proteins).

We purified and crystallized BrxR as described in 'Methods' and determined its structure to $2.3 \AA$ resolution (Figure 4). The protein forms a symmetrical homodimer comprising two intertwined protein chains, each of which contain an N-terminal wHTH domain, a central WYL domain, and a C-terminal WCX domain (consistent with bioinformatic predictions) (Figure 4a,b). The two exposed helices of the wHTH domain (a fold often associated with DNA binding) are positioned and spaced appropriately to interact with DNA at positions separated by approximately one full turn of a B-form double helix. The pair of beta strands that form the "wing" in this domain could also be positioned to contact DNA, as has been shown for other proteins of this class (44). The WYL domains are domain-swapped within the 
homodimeric structure of the protein, with each domain closely associated with the other subunit's underlying $\mathrm{wHTH}$ domain. The core of the homodimer is further stabilized by interaction between helices in each protein subunit that are located between the wHTH and WYL domains (residues 71-119; gray in Figure 4a).

Inspection of the BrxR surface directly opposite its wHTH domains (Figure $4 \mathbf{c , d , e}$ ) revealed a pair of symmetry-related, basic pockets flanked in part by conserved residues from the WYL domain (including S140, Y138, H147, R149, R162, and R182). The pocket and its conserved residues corresponds to the same region that is predicted to mediate cofactor binding in the WYL domain of the Pafbc transcription factor (24). The C-terminal end of one BrxR subunit (ending at amino acid 291) inserts into the pocket of the WYL domain of the opposite subunit (Figure 4e). The electron density for that C-terminal tail is weak (and entirely unobservable for the same C-terminal residues in the opposing subunit), indicating that this region is flexible and partially disordered, and could potentially be displaced by the binding of an effector molecule.

The structure of BrxR suggested that it might act as a DNA-binding protein, and potentially act as a transcriptional regulator. If so, given its structural symmetry it would likely bind a symmetric or nearly symmetric DNA target associated with the BREX operon. To test this possibility, we generated a panel of 13 DNA targets (mostly focused on upstream regions of BREX ORF's) each 250-300 bp in length, and tested the ability of purified BrxR to bind them in electrophoretic mobility shift assays (EMSAs or 'gel shifts'). We observed low affinity interactions to all of those DNA probes that appeared to correspond to non-specific binding (Supplementary Figure S5).

Further examination of the Acinetobacter genomic sequence upstream of the BrxR ORF revealed the presence of a $\sim 70$ basepair sequence that was strongly predicted to form an extended stem loop structure (Figure 5a). This region included a 25 basepair sequence containing a pseudo-palindromic sequence located at the tip of that predicted structure, that overlapped with a predicted bacterial promoter region (Figure 5b) suggested by the BPROM server (45). We therefore tested a $\sim 275$ basepair DNA sequence containing this region in additional EMSAs and observed much higher affinity binding and formation of a unique shifted protein-DNA complex by BrxR, indicative of sequence-specific interaction (Figure 5, inset). Titration of the BrxR protein indicated a dissociation constant of approximately $100 \mathrm{nM}$. We ultimately identified a 35 basepair region in which binding could be observed (corresponding to basepairs -81 to -46 , upstream of BrxR's start codon). This region includes the pseudo-palindromic sequence described above, that is conserved in 9 out of 10 positions between two half-sites spaced 5 nucleotides apart.

We co-crystallized of BrxR with this palindromic sequence and solved a second structure of BrxR bound to the DNA target at $2.3 \AA$ resolution (Figure 6a). The cocrystal structure illustrates that BrxR binds the DNA target through extensive interactions between each subunit's wHTH domain and each 
corresponding target half-site. Each protein subunit and DNA half-site form an interface that buries approximately $870 \AA^{2}$ of surface area, via the formation of up to 36 direct atomic contacts (18 of which are consistent with sequence-specific hydrogen-bonds) by each subunit (analysis facilitated and visualized with the DNAProDB online tool (46)). The DNA duplex maintains a B-form topology and all the bases are found in Watson-Crick interactions with their partners from the opposing complementary strand. The DNA is bent by approximately 10 to $15^{\circ}$ within the plane of the protein-DNA complex, and the minor groove is significantly narrowed across the central five base pair sequence that separates the two related target half-sites.

BrxR makes base-specific contacts to a total of 7 bases in each half-target of DNA (Figure $6 \mathbf{b}$ ). The H3 helices from both HTH domains are positioned in adjacent major grooves such that all base-specific contacts are contributed by a cluster of amino acids at the N-terminal region of the long H3 helix: S37, R38, Q39, Q40 and S42 (Figure 6c). Those residues are highly conserved among BrxR homologs that otherwise display as low as 38\% homology, suggesting that that BrxR homologs may bind to the similar DNA targets in other organisms. Numerous additional contacts (i.e. to the minor groove and phosphoribose backbone) are contributed by residues that form the "wing" in the wHTH domain, including H59, P61, S62, K64, Y66. Overlay of the DNA-bound protein structure to the unbound form of BrxR shows a near perfect match in structure (Supplementary Figure S6), indicating that the unbound form of $\mathrm{BrxR}$ is in the proper configuration for binding this particular sequence. We speculate (see discussion) that cofactor binding to the WYL region of BrxR may induce a structural change in BrxR that could facilitate release from the binding site identified here, and/or enhance binding to a different target sequence than that identified in our biased approach described above.

\section{Deletion of the BrxR gene affects bacterial growth and phage restriction differently than}

disruption of the BrxR gene. Based on the structural and biochemical analyses described above, we generated two additional disruptions to BrxR in the pACYC184-BREX vector and validated them by sequencing across the entire plasmid: a premature stop codon (created by mutating a guanine base at position 62 to adenine, resulting in a stop at codon 21) or a point mutation (R47A) in the wHTH DNA binding surface predicted to interfere with DNA binding. The R47A amino acid substitution was also incorporated into the recombinant protein expression vector, and the corresponding construct was purified (Figure 7a) and examined to ensure that the mutation still resulted in soluble homodimeric protein (Figure 7b), did not cause a significant destabilization of the protein fold (Figure 7c) and eliminated site-specific DNA binding as predicted (Figure 7d). As a further control for experiments examining the effect of R47A on BrxR behavior and function, several additional point mutations were introduced into the WYL domain of the protein; the resulting constructs were individually purified, and their solution behavior and stability characterized (Supplementary Figure S7a-d). One construct from that series (R149A, which is conserved across WYL domains and protein) was shown to still bind the protein's target site similarly to the wild-type protein (Supplementary Figure S7e). 
Whereas the precise deletion of the BrxR gene from the BREX operon significantly reduced phage restriction (Figure 3 and Supplementary Figure S4), cells transformed with the BREX operon harboring displayed similar abilities to restrict $\lambda_{\text {vir }}$ in plaque formation assays (Figure 7e).

Finally, we examined protein expression in E. coli cells in the presence and absence of the BREX operon, which itself harbored either an intact copy of the BrxR coding sequence or a deletion or a disruption of that gene. Polyacrylamide gel electrophoresis (PAGE) analyses (Figure 7f) indicated the visible expression of at least one additional protein from cells harboring the wild-type BREX operon, corresponding to a molecular weight similar to $\mathrm{BrxC}(140 \mathrm{kD})$ or possibly PgIX (134 kD). The expression of that protein was significantly increased in cells that contained a precise deletion of the BrxR gene. Additional control lanes, corresponding to cells that harbored either a clean deletion of $\mathrm{BrxC}$ or an epitope-tagged version of BrxC, implicated that protein as the BREX factor being signficiantly upregulated in the absence of the BrxR gene.

In contrast, cells harboring the BREX operon with a premature stop codon in BrxR displayed little difference in protein expression levels as compared to those harboring the wild-type BREX operon. This appears to reflect our earlier observation that disruption of the BrxR gene via the same stop codon (rather than precisely deleting the gene) had little measurable effect on phage restriction.

\section{DISCUSSION}

\section{The distribution and diversity of WYL proteins within bacterial defense islands and BREX}

systems. The BrxR protein family is clearly widespread. A previously described bioinformatic analysis of PafBC, a bacterial transcriptional regulator involved in DNA damage response that contains a similar HTH-WYL-WCX domain organization as BrxR, identified over 10,000 homologues that are distributed across actinobacteria, proteobacteria, firmicutes and bacterioidetes (24). Similarly, our own BLASTP search (47) using the Acinetobacter $394 \mathrm{BrxR}$ sequence ('BrxR $\mathrm{Acin}_{\text {') }}$ as a starting query returns over 9000 hits with sequence identities of approximately $20 \%$ or higher (corresponding to E-values of 28 or better). BrxR homologs spanning a wide range of sequence identities (as low as 25\%) are frequently linked to defense systems across a wide range of bacterial phyla, indicating a strong selective pressure to maintain BrxR association with these islands. A significant fraction of those BrxR homologues are found within gamma proteobacteria (which includes enterobacteria such as Escherichia fergesonii, Vibrionaceae including Vibrio cholera, and Pseudomonacaea including the Pseudomonas genera). However, there are numerous BrxR homologues found in alternative bacterial clades and genera, which one might expect for a regulatory protein frequently associated with mobile genetic islands and their corresponding phage defense and antibiotic resistance systems. 
We constructed a multi-sequence alignment (Figure 8) from a sampling of nine BrxR sequences spanning amino acid identities (relative to BrxR Acin $_{\text {) }}$ ranging from 92\% (Klebsiella) to 22\% (Escherichia fergesonii, which has been similarly characterized (48)). Residues involved in contacting individual base pairs in the BrxR $R_{A c i n} D N A$ target site (spanning a motif corresponding to $S_{37} R_{38} Q_{39} Q_{40} S_{42}$ in $B r x R_{A c i n}$; Figure 4) are conserved among homologues that exhibit down to $40 \%$ amino acid identity relative to $B_{\text {BrxR }}$ Acin. In comparison, BrxR from Escherichia fergesonii (which recognizes a different pseudopalindromic target site in the same region upstream of its own BREX operon) is diverged across those same residues. Overall, 64 residues are strictly conserved across the entire range of homologues shown in our alignment; of those, a cluster of residues correspond to a conserved basic pocket in the WYL domain (illustrated in Figure 6).

The BrxR gene and BrxR protein appear to play unique cis- and trans-regulatory roles. The observation that the effect of a precise deletion of the BrxR coding sequence from the BREX operon (which resulted in toxicity and a reduction in phage restriction) differed from the negligible effect of an early stop codon in the BrxR coding sequence (that otherwise left the BrxR gene intact) was unexpected. This result was reproduced in a parallel experiment in which a single amino acid mutation (R47A) abrogated BrxR binding to its DNA target. These results imply that in the Acinetobacter 394 BREX system, the BrxR gene itself may contain additional cis regulatory elements, such as an alternative promoter, that when removed disrupt the normal expression of BREX ORF's, and in turn the normal restriction function of BREX (Figure 3). Consistent with this possibility, an analysis of the BrxR coding sequence from Acinetobacter using the BPROM server (45) suggests the possible presence of a promoter within the BrxR gene.

The toxicity of E. coli harboring the BREX operon with a precise deletion of the BrxR ORF (' $\Delta \mathrm{BrxR}$ '), as well as those with deletions of the BrxB or PglZ genes, may be caused by an imbalance of proteins that form toxin-antitoxin pairs. PgIX and PgIZ have previously been implicated as toxin-antitoxin partners, and it has been postulated that BREX may contain additional similar interactions (16). The marked increase in $\operatorname{BrxC}$ protein levels that we observe in $\triangle \mathrm{BrxR}$ cells relative to cells expressing an intact $B R E X$ operon (Figure 7F) may implicate BrxC as being such a toxin (although we do not know its antitoxin partner).

Potential regulation of BrxR activity. The observation that BrxR binds to a specific DNA target upstream of the BREX operon at a position that overlaps with a predicted bacterial promoter (Figure 5) indicates that it is likely a transcriptional regulator. However, the presence of a highly conserved region in BrxR's WYL domain further implies that it may be involved in additional layers of regulation that depend upon the binding of a second messenger or a phage-derived factor that is produced during a phage challenge. The binding of such a molecule might alter the relative binding affinity of BrxR towards competing DNA targets, as has been previously hypothesized for the PafBC regulator as part of a bacterial DNA damage response $(23,24)$. Unlike PafBC, which appears to require a significant domain 
rearrangement to bind DNA (thought to be driven by binding of an unknown effector to the WYL domain), the BrxR protein visualized in this study is preorganized in a homodimeric conformation that is appropriate for target binding with negligible conformational changes to the protein architecture. Therefore, any regulated changes in DNA binding specificity for BrxR might be expected to involve relatively subtle structural rearrangements.

The published literature provides a number of anecdotal lines of evidence that proteins containing WYL domains might act as transcriptional regulators that display differential activities (involving alteration of binding activities and specificities, and/or transitioning between repressive or stimulatory functions) as part of a corresponding defense system's response to a foreign invader or challenge (21). For example, a WYL domain protein (sll7009) appears to act as a transcriptional repressor of a CRISPR-Cas system in Synechocystis sp (22), and WYL proteins have been shown to regulate other CRISPR systems (49). Two recent studies both demonstrate that WYL domain proteins found in BREX systems associated with highly mobile SXT Integrative and Conjugative Element ('SXT ICE') defense islands, in Vibrio cholerae and Proteus mirabilis respectively, appear to play important regulatory roles in BREX action (19, 20). Our own alignment of WYL domains from these elements (which share $~ 25 \%$ sequence identity with BrxR $_{\text {Acin }}$ ) and subsequent structural modeling carried out with the AlphaFold server (50) (Supplementary Figure S8) indicates that those proteins also share the same wHTH-WYL-WCX domain organization and appear to adopt a conformation of those domains that is seen in our crystal structures of the $B_{r x R} R_{A c i n}$ homodimer.

The presence of the WYL domain in $B_{x x R_{A c i n}}$ might confer a sensitivity to an effector molecule (perhaps a bacterial second messenger or a phage-associated factor) as part of an early response to a phage challenge. If true, it is interesting to speculate on the nature of such an effector and the possibility that such an effector might modulate BrxR regulation of BREX activity. The highly conserved pockets in the WYL domain found across BrxR homologues (Figure 6cde and Figure 8) appear to be positively charged, which could be compatible with binding to a nucleotide-derived ligand. Those pockets are loosely associated with the final few residues of the WCX domain's C-terminal tail, which might be displaced upon effector binding. As well, two adjoining a-helices in the WCX domains, that are immediately upstream of their C-termini (residues 253-263) partially occlude a considerably longer highly basic channel that connects the conserved pockets in the two WYL domains; if also displaced a surface of sufficient area and dimensions to bind a larger moiety (such as a DNA or RNA oligonucleotide) would be exposed. Consistent with this latter possibility, other proteins have been observed to use WYL and WCX domains to bind DNA or RNA effectors $(24,51-55)$.

Potential functions of BrxR as a BREX regulator. The observation that an early stop codon in BrxR

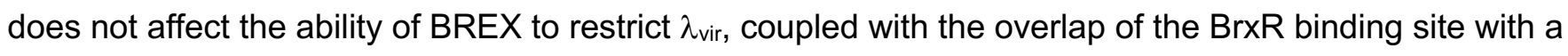
promoter in its upstream region, suggests that BrxR may function as a repressor of the BREX system. 
Such a role has been proposed for other WYL protein-mediated regulation of defense systems $(19,22)$. We speculate there may be either (or both) of two potential roles for an inhibitory function of BrxR:

1) Function as a "2nd line of defense". Activation of the BREX system in its native context may require release from a repressed state mediated by BrxR. However, such a mechanism poses a possible condundrum for the bacteria: if cells were to rely on BREX as a 'front line' defense, the time required for the system to emerge from a repressed state might be too slow relative to the initial appearance and action of phage. Perhaps instead, BREX might provide a 'second line' of defense that is activated during the time that first responders (such as restriction-modification and CRISPR systems) to successfully ward off an initial challenge. The BREX system would then be primed and ready if resistant phage break through these front lines of defense. One might imagine that a rare phage variant with appropriate resistance factors (perhaps present at very low levels in the initial phage population, and then selected for during the first early stages of a challenge) might eventually create a 'second wave' requiring additional strategies for restriction.

2) Facilitate horizontal transfer into new hosts. It is notable that in various bacterial systems (such as Vibrio cholera (19), Proteus mirabilis (20) or Escherichia fergesonii (56)), BrxR homologues are coupled to a wide range defense systems (including BREX) that are contained within mobile elements, suggesting that these defense systems frequently transfer between hosts. We postulate that BrxR may transiently suppress a BREX-mediated toxic activity during horizontal gene transfer so that PgIX (and associated factors) has time to methylate and protect the new host's genome. Such a role has been described for C-proteins during horizontal transfer of R-M systems $(12,13)$. 


\section{Acknowledgements}

We thank Dr. Harmit Malik for helpful discussions and for support of KF and DH. Work performed by KF and $\mathrm{DH}$ was further supported by a grant from the Mathers Foundation and by the HHMI. Support was was also provided New England Biolabs and by the NIH for both BLS (R01 GM105691) and BKK (R15 GM140375). The Berkeley Center for Structural Biology is supported in part by the National Institutes of Health, National Institute of General Medical Sciences, and the Howard Hughes Medical Institute. The Advanced Light Source is supported by the Director, Office of Science, Office of Basic Energy Sciences, of the U.S. Department of Energy under Contract No. DE-AC02-05CH11231. The Pilatus detector was funded under NIH grant S10OD021832. The ALS-ENABLE beamlines are supported in part by the National Institutes of Health, National Institute of General Medical Sciences, grant P30 GM124169

\section{DATA AVAILABILITY}

The structures described in this manuscript have been deposited in the RCSB protein database (PDB ID codes 7T8K and 7T8L). The original source data and raw images corresponding to the biochemical analyses BREX and BrxR function and activity have been uploaded to the Harvard Dataverse public repository and are also available upon request from the authors.

\section{CONFLICT OF INTEREST STATEMENT}

YL and RDM are employees of New England Biolabs, a for-profit biotech company that manufactures enzymes and reagents for commercial sale and was the original source of the BREX system described in this manuscript. BLS is a paid consultant for New England Biolabs, which also funded this work in his laboratory. 


\section{References}

1. Blower TR, Salmond GP, Luisi BF. Balancing at survival's edge: the structure and adaptive benefits of prokaryotic toxin-antitoxin partners. Curr Opin Struct Biol. 2011;21(1):109-18.

2. Makarova KS, Wolf YI, Snir S, Koonin EV. Defense islands in bacterial and archaeal genomes and prediction of novel defense systems. J Bacteriol. 2011;193(21):6039-56.

3. Bernheim A, Sorek R. The pan-immune system of bacteria: antiviral defence as a community resource. Nat Rev Microbiol. 2020;18(2):113-9.

4. Chopin MC, Chopin A, Bidnenko E. Phage abortive infection in lactococci: variations on a theme. Curr Opin Microbiol. 2005;8(4):473-9.

5. Gao L, Altae-Tran H, Bohning F, Makarova KS, Segel M, Schmid-Burgk JL, et al. Diverse enzymatic activities mediate antiviral immunity in prokaryotes. Science. 2020;369(6507):1077-84.

6. Koonin EV, Makarova KS, Wolf YI. Evolutionary Genomics of Defense Systems in Archaea and Bacteria. Annu Rev Microbiol. 2017;71:233-61.

7. Labrie SJ, Samson JE, Moineau S. Bacteriophage resistance mechanisms. Nat Rev Microbiol. 2010;8(5):317-27.

8. Goldfarb T, Sberro H, Weinstock E, Cohen O, Doron S, Charpak-Amikam Y, et al. BREX is a novel phage resistance system widespread in microbial genomes. EMBO J. 2015;34(2):169-83.

9. Gordeeva J, Morozova N, Sierro N, Isaev A, Sinkunas T, Tsvetkova K, et al. BREX system of Escherichia coli distinguishes self from non-self by methylation of a specific DNA site. Nucleic Acids Res. 2019;47(1):253-65.

10. Chinenova TA, Mkrtumian NM, Lomovskaia ND. [Genetic characteristics of a new phage resistance trait in Streptomyces coelicolor A3(2)]. Genetika. 1982;18(12):1945-52.

11. Sumby $\mathrm{P}$, Smith MC. Genetics of the phage growth limitation (Pgl) system of Streptomyces coelicolor A3(2). Mol Microbiol. 2002;44(2):489-500.

12. Mruk I, Blumenthal RM. Real-time kinetics of restriction-modification gene expression after entry into a new host cell. Nucleic Acids Res. 2008;36(8):2581-93.

13. Williams K, Savageau MA, Blumenthal RM. A bistable hysteretic switch in an activator-repressor regulated restriction-modification system. Nucleic Acids Res. 2013;41(12):6045-57.

14. Ye Q, Lau RK, Mathews IT, Birkholz EA, Watrous JD, Azimi CS, et al. HORMA Domain Proteins and a Trip13-like ATPase Regulate Bacterial cGAS-like Enzymes to Mediate Bacteriophage Immunity. Mol Cell. 2020;77(4):709-22.e7.

15. Hoskisson PA, Smith MC. Hypervariation and phase variation in the bacteriophage 'resistome'. Curr Opin Microbiol. 2007;10(4):396-400.

16. Hoskisson PA, Sumby P, Smith MCM. The phage growth limitation system in Streptomyces coelicolor $A(3) 2$ is a toxin/antitoxin system, comprising enzymes with DNA methyltransferase, protein kinase and ATPase activity. Virology. 2015;477:100-9.

17. Laity C, Chater KF, Lewis CG, Buttner MJ. Genetic analysis of the phi C31-specific phage growth limitation (Pgl) system of Streptomyces coelicolor A3(2). Mol Microbiol. 1993;7(2):329-36.

18. Sumby P, Smith MC. Phase variation in the phage growth limitation system of Streptomyces coelicolor A3(2). J Bacteriol. 2003;185(15):4558-63.

19. LeGault KN, Hays SG, Angermeyer A, McKitterick AC, Johura FT, Sultana M, et al. Temporal shifts in antibiotic resistance elements govern phage-pathogen conflicts. Science. 2021;373(6554). 
20. Slattery S, Tony Pembroke J, Murnane JG, Ryan MP. Isolation, nucleotide sequencing and genomic comparison of a Novel SXT/R391 ICE mobile genetic element isolated from a municipal wastewater environment. Sci Rep. 2020;10(1):8716.

21. Makarova KS, Anantharaman V, Grishin NV, Koonin EV, Aravind L. CARF and WYL domains: ligand-binding regulators of prokaryotic defense systems. Front Genet. 2014;5:102.

22. Hein S, Scholz I, Voss B, Hess WR. Adaptation and modification of three CRISPR loci in two closely related cyanobacteria. RNA Biol. 2013;10(5):852-64.

23. Muller AU, Imkamp F, Weber-Ban E. The Mycobacterial LexA/RecA-Independent DNA Damage Response Is Controlled by PafBC and the Pup-Proteasome System. Cell Rep. 2018;23(12):3551-64.

24. Muller AU, Leibundgut M, Ban N, Weber-Ban E. Structure and functional implications of WYL domain-containing bacterial DNA damage response regulator PafBC. Nat Commun. 2019;10(1):4653.

25. Eid J, Fehr A, Gray J, Luong K, Lyle J, Otto G, et al. Real-time DNA sequencing from single polymerase molecules. Science. 2009;323(5910):133-8.

26. Roberts RJ, Vincze T, Posfai J, Macelis D. REBASE--a database for DNA restriction and modification: enzymes, genes and genomes. Nucleic Acids Res. 2015;43(Database issue):D298-9.

27. Altschul SF, Gish W, Miller W, Myers EW, Lipman DJ. Basic local alignment search tool. J Mol Biol. 1990;215(3):403-10.

28. Chang AC, Cohen SN. Construction and characterization of amplifiable multicopy DNA cloning vehicles derived from the P15A cryptic miniplasmid. J Bacteriol. 1978;134(3):1141-56.

29. Ettwiller L, Buswell J, Yigit E, Schildkraut I. A novel enrichment strategy reveals unprecedented number of novel transcription start sites at single base resolution in a model prokaryote and the gut microbiome. BMC Genomics. 2016;17:199.

30. Kechin A, Boyarskikh U, Kel A, Filipenko M. cutPrimers: A New Tool for Accurate Cutting of Primers from Reads of Targeted Next Generation Sequencing. J Comput Biol. 2017;24(11):1138-43.

31. Langmead B, Salzberg SL. Fast gapped-read alignment with Bowtie 2. Nat Methods. 2012;9(4):357-9.

32. Robinson JT, Thorvaldsdóttir H, Winckler W, Guttman M, Lander ES, Getz G, et al. Integrative genomics viewer. Nat Biotechnol. 2011;29(1):24-6.

33. Yan B, Boitano M, Clark TA, Ettwiller L. SMRT-Cappable-seq reveals complex operon variants in bacteria. Nat Commun. 2018;9(1):3676.

34. Bailone A, Galibert F. Nucleotide sequence of the operators of lambda ultravirulent mutants. Nucleic Acids Res. 1980;8(10):2147-64.

35. Doublie S. [29] Preparation of selenomethionyl proteins for phase determination. Methods Enzymol. 1997;276:523-30.

36. Otwinowski Z, Minor W. Processing of X-ray diffraction data collected in oscillation mode. Methods Enzymol. 1997;276:307-26.

37. Ness SR, de Graaff RA, Abrahams JP, Pannu NS. CRANK: new methods for automated macromolecular crystal structure solution. Structure. 2004;12(10):1753-61.

38. Emsley P, Cowtan K. Coot: model-building tools for molecular graphics. Acta Crystallogr D Biol Crystallogr. 2004;60(Pt 12 Pt 1):2126-32.

39. Murshudov GN, Skubák P, Lebedev AA, Pannu NS, Steiner RA, Nicholls RA, et al. REFMAC5 for the refinement of macromolecular crystal structures. Acta Crystallogr D Biol Crystallogr. 2011;67(Pt 4):355-67. 
40. Liebschner D, Afonine PV, Baker ML, Bunkóczi G, Chen VB, Croll TI, et al. Macromolecular structure determination using $X$-rays, neutrons and electrons: recent developments in Phenix. Acta Crystallogr D Struct Biol. 2019;75(Pt 10):861-77.

41. Hui W, Zhang W, Kwok LY, Zhang H, Kong J, Sun T. A Novel Bacteriophage Exclusion (BREX) System Encoded by the pgIX Gene in Lactobacillus casei Zhang. Appl Environ Microbiol. 2019;85(20).

42. Sibley $\mathrm{MH}$, Raleigh EA. Cassette-like variation of restriction enzyme genes in Escherichia coli $\mathrm{C}$ and relatives. Nucleic Acids Res. 2004;32(2):522-34.

43. Fudrini Olivencia B, Müller AU, Roschitzki B, Burger S, Weber-Ban E, Imkamp F. Mycobacterium smegmatis PafBC is involved in regulation of DNA damage response. Sci Rep. 2017;7(1):13987.

44. Aravind L, Anantharaman V, Balaji S, Babu MM, Iyer LM. The many faces of the helix-turn-helix domain: transcription regulation and beyond. FEMS Microbiol Rev. 2005;29(2):231-62.

45. Solovyev V, Salamov A. Automatic Annotation of Microbial Genomes and Metagenomic Sequences. In: Li RW, editor. Metagenomics and its Applications in Agriculture, Biomedicine and Environmental Studies. Happague NY: Nova Science; 2011. p. 61-78.

46. Sagendorf JM, Markarian N, Berman HM, Rohs R. DNAproDB: an expanded database and webbased tool for structural analysis of DNA-protein complexes. Nucleic Acids Res. 2020;48(D1):D277-d87.

47. Database resources of the National Center for Biotechnology Information. Nucleic Acids Res. 2018;46(D1):D8-d13.

48. Picton DM, Harling-Lee JD, Duffner SJ, Went SC, Morgan RD, Hinton JCD, et al. A widespread family of WYL-domain transcriptional regulators co-localises with diverse phage defence systems and islands. submitted. 2021.

49. Zhang H, Dong C, Li L, Wasney GA, Min J. Structural insights into the modulatory role of the accessory protein WYL1 in the Type VI-D CRISPR-Cas system. Nucleic Acids Res. 2019;47(10):5420-8.

50. Varadi M, Anyango S, Deshpande M, Nair S, Natassia C, Yordanova G, et al. AlphaFold Protein Structure Database: massively expanding the structural coverage of protein-sequence space with highaccuracy models. Nucleic Acids Res. 2021.

51. Andis NM, Sausen CW, Alladin A, Bochman ML. The WYL Domain of the PIF1 Helicase from the Thermophilic Bacterium Thermotoga elfii is an Accessory Single-Stranded DNA Binding Module. Biochemistry. 2018;57(7):1108-18.

52. Bertram K, Agafonov DE, Dybkov O, Haselbach D, Leelaram MN, Will CL, et al. Cryo-EM Structure of a Pre-catalytic Human Spliceosome Primed for Activation. Cell. 2017;170(4):701-13.e11.

53. Khusial P, Plaag R, Zieve GW. LSm proteins form heptameric rings that bind to RNA via repeating motifs. Trends Biochem Sci. 2005;30(9):522-8.

54. Santiago-Frangos A, Kavita K, Schu DJ, Gottesman S, Woodson SA. C-terminal domain of the RNA chaperone Hfq drives sRNA competition and release of target RNA. Proc Natl Acad Sci U S A. 2016;113(41):E6089-e96.

55. Vecerek B, Rajkowitsch L, Sonnleitner E, Schroeder R, Bläsi U. The C-terminal domain of Escherichia coli Hfq is required for regulation. Nucleic Acids Res. 2008;36(1):133-43.

56. Picton DM, Luyten YA, Morgan RD, Nelson A, Smith DL, Dryden DTF, et al. The phage defence island of a multidrug resistant plasmid uses both BREX and type IV restriction for complementary protection from viruses. Nucleic Acids Res. 2021;49(19):11257-73. 


\section{Figure Captions}

Figure 1. Type I BREX operon from Acinetobacter strain NEB394. Panel a: Seven genes within the BREX locus encode for proteins ranging in size from $22 \mathrm{kD}$ (BrxB, 191 residues) to $134 \mathrm{kD}$ (PgIX, 1173 residues). A transcription start site analysis shows that BREX is expressed via a single long transcript (upper arrow and dashed line) in logarithmically grown Acinetobacter394 cells in the absence of a phage challenge. Panel b: PacBio sequencing of Acinetobacter NEB394 and E. coli genomes (harboring the endogenous BREX operon and the same operon introduced via transformation with plasmid pACYCBREX) identifies the host target site and base (5'-GTAGAT-3') methylated by the BREX system via action of the PgIX methyltransferase. Panel c: Systematic deletion of each BREX gene from the pACYC-BREX plasmid and additional PacBio methylation analysis in transformed $E$. coli cells demonstrates that four BREX genes (BrxA, BrxB, BrxC and $\mathrm{PgIZ}$ ) are required for genome modification in addition to the PgIX methyltransferase. Panel d: Growth curves of NEB2683 E. coli cells transformed with PACYC-BREX plasmids in which each BREX gene has been systematically deleted. Three deletions ( $\triangle \mathrm{PglZ}, \Delta \mathrm{BrxB}$ and $\Delta \mathrm{BrxR}$ ) appear to display lags or reductions in growth rates. These observations are reflected in reduced transformation efficiencies and small or irregular colony size for the same constructs (Supplementary Figure S1).

Figure 2. BREX is active in E. coli preceded either by a constitutive tet promoter or by the upstream genomic sequence from Acinetobacter. Panel a: BREX transcription start site analysis in Acinetobacter 394. A single long transcript is detected beginning 23 base pairs upstream of the BrxR start codon. The top track shows BREX ORFs. The bottom track shows PacBio SMRT capable seq showing coverage (gray). This shows a strong TSS just before BrxR and the BREX operon, with very low levels of internal operon reads, indicating one TSS for the entire operon. See Supplementary Figure S2 for additional detail. Panel b: Restriction by BREX visualized in a plaque formation assay. Restriction was assayed using a plaque formation assay with $\lambda_{\text {vir }}$ phage deployed against $E$. coli strain ER2683. For each construct, serial 10-fold dilutions of phage were spotted on a bacterial lawn, and individual plaques were counted. All assays were repeated in biological triplicate. The intact BREX operon generates similar (30- to 50-fold) reductions in plaquing efficiency, relative to the empty PACYC vector, when preceded by either a constitutive tet promoter ('tet') or by the upstream putative promoter and regulatory region from Acinetobacter ('native'). Panel c:). Restriction by the same BREX constructs in liquid culture. Bacterial cultures were challenged with $\lambda_{\text {vir }}$ phage at the indicated $\mathrm{MOI}$ and culture density $\left(\mathrm{OD}_{600} \mathrm{~nm}\right)$ was monitored over time. 
Figure 3. BREX restriction when each gene is precisely deleted. Panel a: Fold change in phage plaque formation using $\lambda_{\text {vir }}$ phage and $E$. coli strain ER2683 as a function of the presence or absence of each gene in the BREX operon. Cells transformed with the indicated pACYC constructs, growing at log phase, were mixed with $0.5 \%$ top agar, plated on chloramphenicol plates to form a lawn, then spotted with 10-fold serial dilutions of $\lambda_{\text {vir }}$ phage. The plaque formation efficiency of $\lambda_{\text {vir }}$ phage on a 'cells only' (no BREX) control is normalized to 1 ; the corresponding fold reduction in plaquing formation efficiency of the same phage is then indicated for WT BREX and for BREX harboring a precise deletion of each gene. All seven genes appear to be involved in BREX restriction function. Panel b: Growth of $E$. coli strain ER2683 (New England Biolabs) challenged with the $\lambda_{\text {vir }}$ phage at an MOI of 1.0. The intact BREX system conferred robust protection against $\lambda_{\text {vir }}$ phage, whereas individual deletions of each BREX gene led to a significant reduction in phage restriction at both high $\mathrm{MOI}$ (shown here) and a lower $\mathrm{MOI}$ of approximately 0.01 (Supplementary Figure S4).

Figure 4. Crystal structure of BrxR. Top: Domain boundaries of BrxR. Residues 71-119 (gray) are involved in homodimer formation. Panel a: Topology of one subunit of the BrxR dimer, colored as shown in the top schematic. Panel b: Structure of the BrxR homodimer, with one subunit colored as a spectrum ranging from the blue $\mathrm{N}$-terminus to the red $\mathrm{C}$-terminus and the second subunit colored in dark teal for contrast. The protein forms an intertwined, domain swapped homodimer. Panels $\boldsymbol{c}$ and $\boldsymbol{d}$ : Top-down views, respectively as a ribbon diagram and a charged electrostatic rendering, of the protein surface opposite the wHTH domains. The WYL domains (green in panel c) are at opposite ends of the protein and form a pair of positively charged pockets (blue color in panel d). Bound cryoprotectant ethylene glycol molecules observed in the interface between protein subunits and in the WYL pockets are indicated with yellow sticks (panel c). The boxes outline the WYL domain and its pocket illustrated in panel e. Panel e: Close up view of the pocket formed by a WYL domain and multiple conserved residues in that region. Density for the C-terminus (yellow) is weak in the site shown in the panel and is not observed in the opposing site, indicating high flexibility for that portion of the protein.

Figure 5. Sequence, predicted secondary structure and BrxR binding site upstream of the BREX operon. Panel a: The Acinetobacter genomic sequence immediately upstream of BrxR ORF, spanning approximately 97 basepairs, contains an $\sim 70$ basepair sequence that is strongly predicted to form an extended stem loop structure, including a 25 basepair sequence corresponding to a pseudo-palindrome located at the tip of the predicted hairpin (indicated by bracket). When presented to BrxR as a doublestranded DNA construct (red and grey bases), electrophoretic mobility shift binding assays demonstrate binding with a dissociation constant of approximately $100 \mathrm{nM}$. The imperfect palindrome formed by the sequence is conserved between half-sites at 9 out of 10 positions, with the half-sites spaced 5 nucleotides apart. Grey arrow illustrates the transcription start site for the operon; black arrow depicts the translational start site for BrxR. Panel b: The BrxR binding site overlaps with a predicted bacterial 
promoter (purple; identified using the BPROM server (45)) that contains a canonical -35 box (undlerlined upper case bases) and downstream -10 box motif.

Figure 6. Crystal structure of BrxR bound to DNA target site. Panel a: Structure of the BrxR homodimer bound to the DNA target shown in Figure 4. The conformation of the DNA-bound protein is closely superimposable with the unbound protein (Supplementary Figure S6) with an overall rmsd across all $\alpha$-carbons of approximately $0.8 \AA$, indicating that the apo-protein is in the proper conformation to interact with this target sequence. Panel $\boldsymbol{b}$ : The DNA contacting surface formed by each protein subunit's wHTH domain. Base-specific contacts are labeled in black and include a group of positively charged and polar residues spanning S37, R38, Q39, Q40 and S42, which are part of a conserved SRQQXS sequence motif conserved across the closest homologues of Acinetobacter BrxR. Nonspecific contacts to the DNA backbone and additional basic residues that contribute to overall affinity are labeled in grey and include R11, K46, R47, K64, H59 and Y66. Panel c: Contacts between BrxR and the bound DNA target site extend across 11 bases for each DNA half-site. Figure panel generated using the DNAProDB online analysis tool (46).

\section{Figure 7. Characterization of a BrxR point mutant with reduced DNA target binding affinity.}

Panels a-c: BrxR containing a single point mutation in its DNA binding domain (R47A) was expressed and purified to homogeneity; it behaves similarly to the wild-type protein dimer in solution as indicated by its elution profile on size exclusion chromatography (SEC) and displays similar thermal stability and unfolding behavior as the wild-type protein. Panel $\boldsymbol{d}$ : Electrophoretic mobility shift assays (EMSA) demonstrate a significant reduction in DNA target binding by purified BrxR (R47A) protein. Panel e: Phage restriction plaque assays indicate that unlike the effect of a precise deletion of the BrxR gene (Figure 3; which causes significant toxicity and reduction in BREX restriction activity), the disruption of the BrxR gene by incorporation of an early stop codon or by introduction of a mutation that blocks BrxR DNA binding activity has little effect on cell growth or phage restriction in the same assays. Panel $f$ : SDS PAGE analysis of whole cell lysates of E. coli ER2683 cells transformed with the following pACYC-BREX constructs: cells only (lane 1); pACYC vector only (lane 2); WT BREX (lane 3); a precise deletion of the BrxR gene ( $\triangle \mathrm{BrxR}$; lane 4); an epitope-tagged version of $\mathrm{BrxC}$ that runs slightly higher than untagged BrxC (BrxC-TST; lane 5); a precise deletion of the $\mathrm{BrxC}$ gene ( $\triangle \mathrm{BrxC}$; lane 6$)$; BREX expressed from its native promoter (lane 7). Transformation with pACYC harboring wild-type BREX results in the appearance of a novel band at approximately $150 \mathrm{kD}$ (arrow), and deletion of the BrxR gene results in significant increase in its intensity (compare lanes 3 and 4). The same band disappears altogether when BrxC gene is deleted from the BREX operon (lane 6), and shifts slightly upward when BrxC is fused to a 35 amino acid twin-strep tag (lane 5), indicating that the upregulated protein product is BrxC. This same band is present in when the BREX operon is expressed from the native promoter (lane 7), albeit at a reduced level compared to when BREX is expressed from the constitutive tet promoter (lane 3). 
Figure 8. Multi-sequence alignment of BrxR homologues. Residue coloring indicates the domains of the protein as similarly colored in Figure 4. Residues that are entirely conserved across a wide range of identities relative to $\mathrm{BrxR}_{\mathrm{Acin}}(22 \%$ overall identity) are indicated with bold font and asterisks. Residues that make contacts to individual DNA bases in the target site (Figure 6) and that are conserved for homologues down to approximately $40 \%$ overall sequence identity are highlighted. Residues that comprise the conserved core of the WYL domain are indicated with a box (also indicated in Figure 4). 


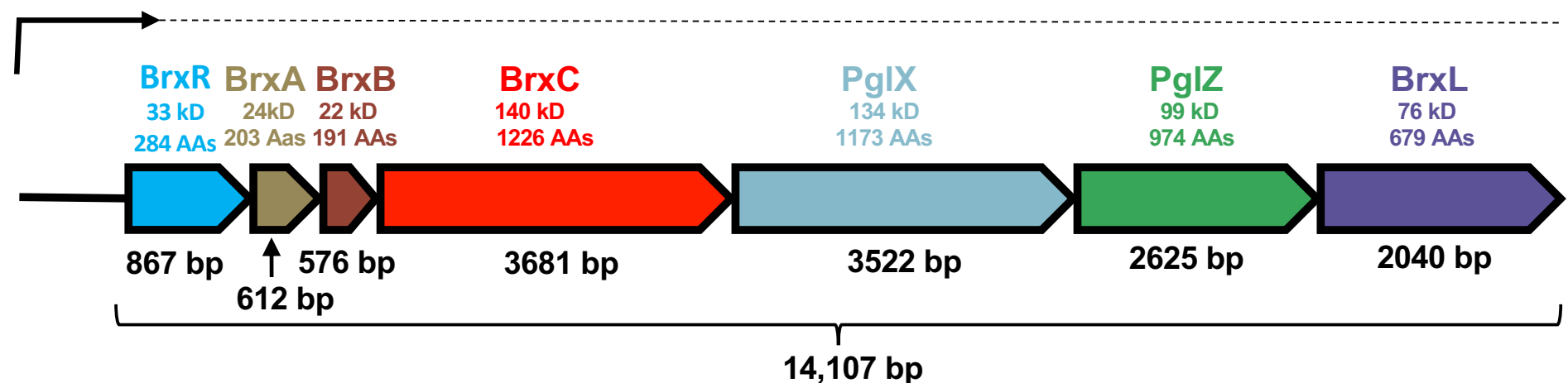

b

\begin{tabular}{|rcccccc|}
\hline \# of sites with IPD $>$ = 2: & 0 & 0 & 0 & 0 & 1317 & 0 \\
\% of sites with IPD >= 2: & 0.0 & 0.0 & 0.0 & 0.0 & 99.8 & 0.0 \\
average IPD: & 1.0 & 1.0 & 1.0 & 1.0 & 5.7 & 1.0 \\
& $\mathbf{G}$ & $\mathbf{T}$ & $\mathbf{A}$ & $\mathbf{G}$ & $\mathbf{A}$ & $\mathbf{T}$ \\
& $\mathbf{C}$ & $\mathbf{A}$ & $\mathbf{T}$ & $\mathbf{C}$ & $\mathbf{T}$ & $\mathbf{A}$ \\
average IPD: & 1.0 & 1.0 & 1.0 & 1.0 & 1.0 & 1.0 \\
\% of sites with IPD $>=2:$ & 0.2 & 0.0 & 0.0 & 0.0 & 0.0 & 0.1 \\
\# of sites with IPD $>=2:$ & 2 & 0 & 0 & 0 & 0 & 1 \\
\hline
\end{tabular}

C

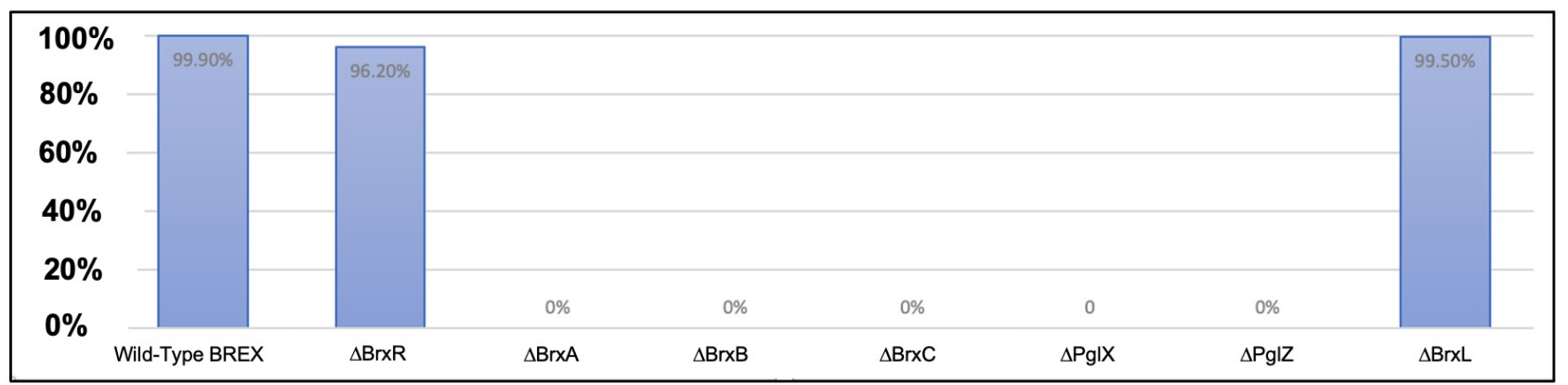

d
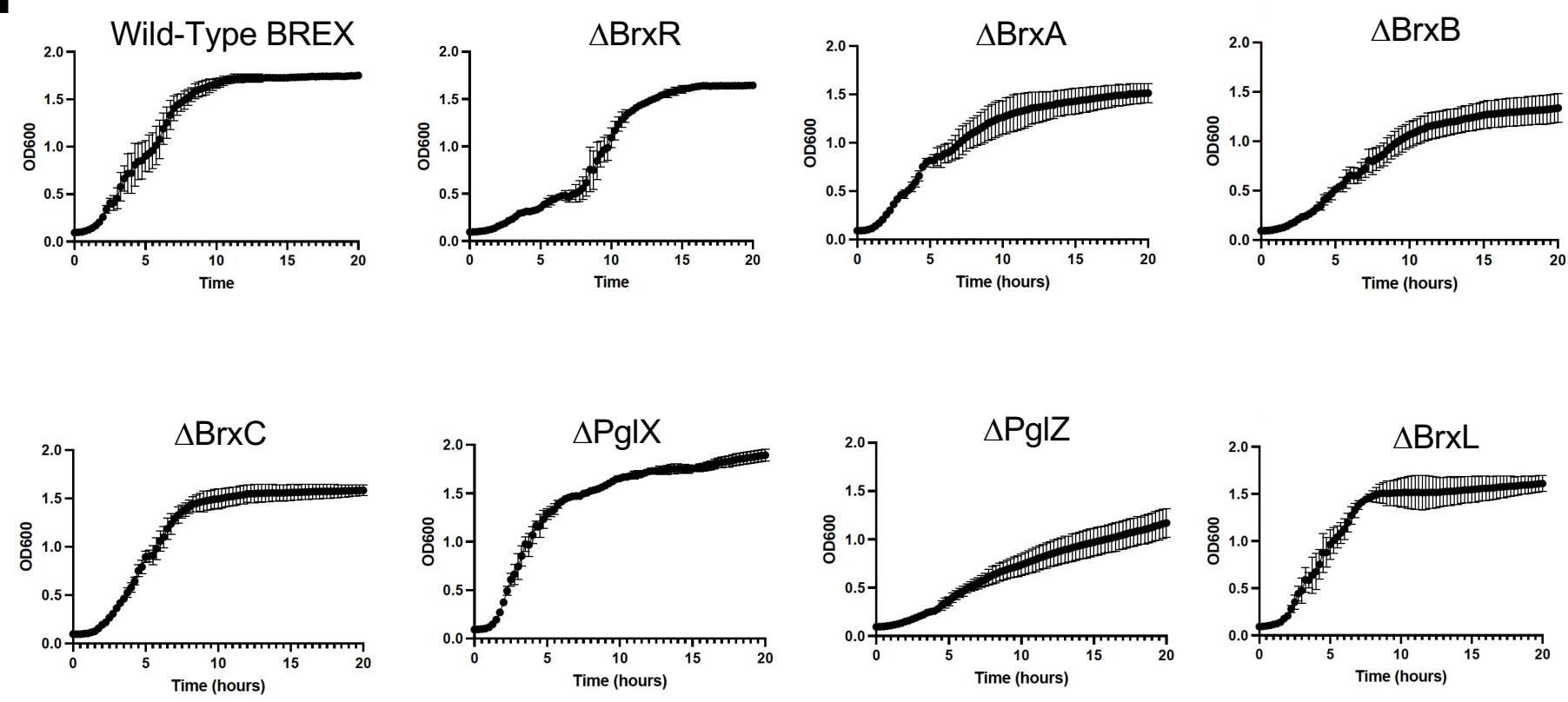


\section{a}

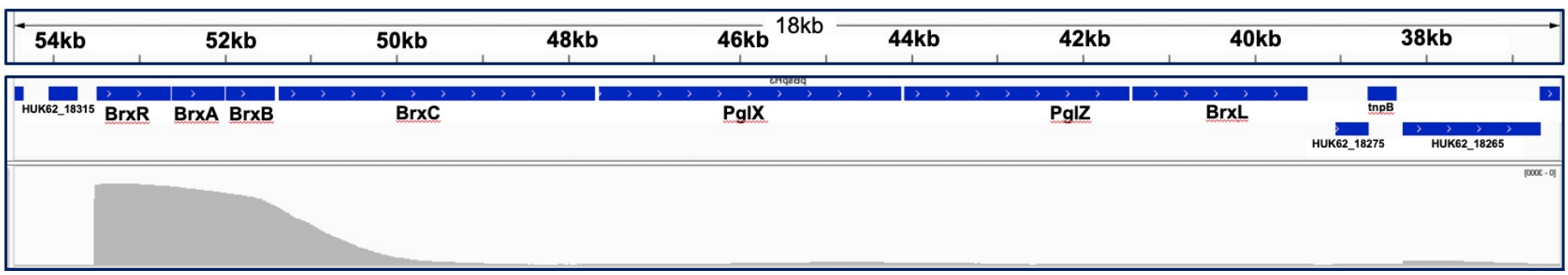

b
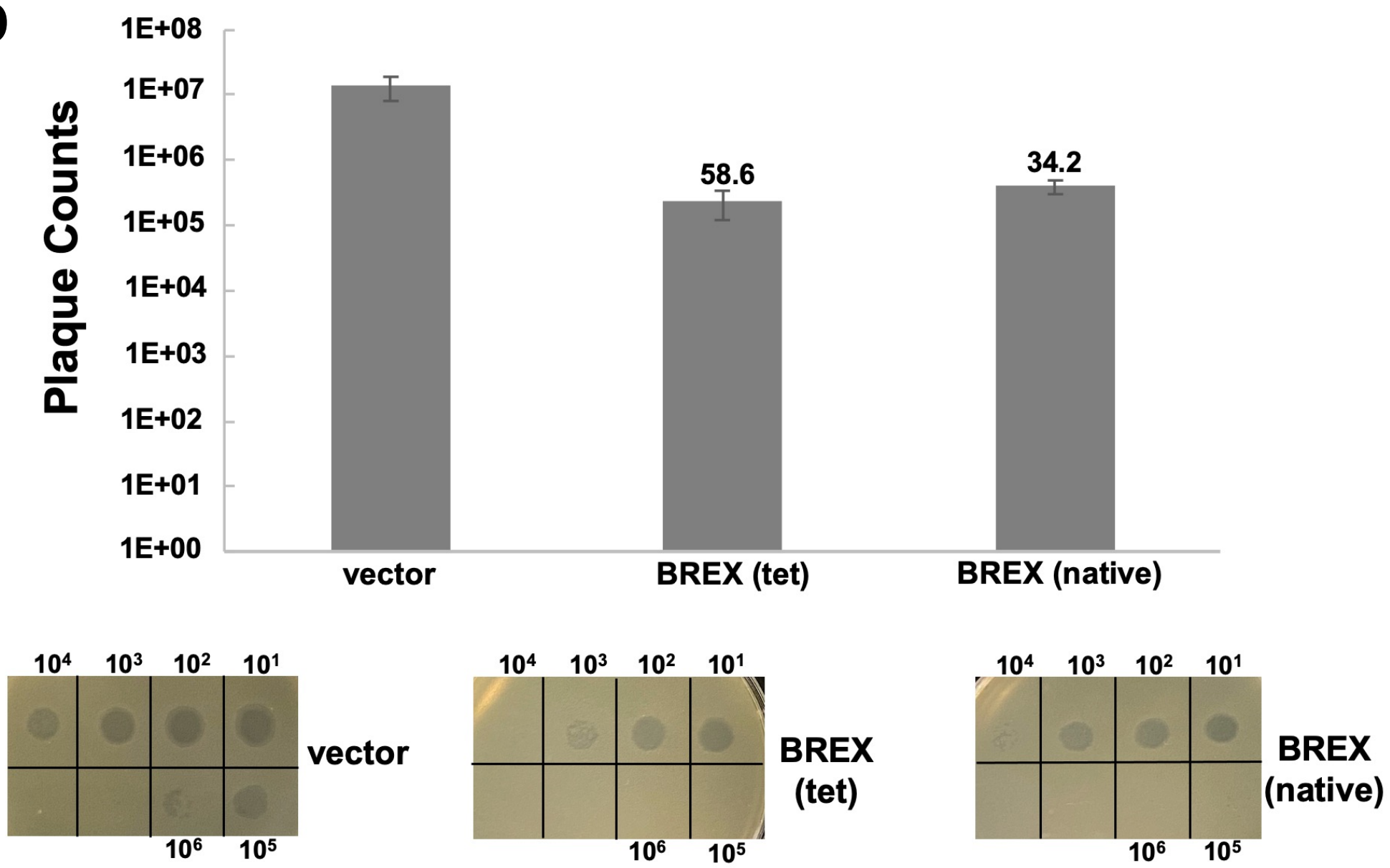

MOI 0

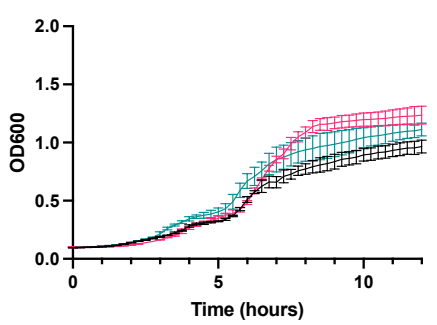

MOI 0.001

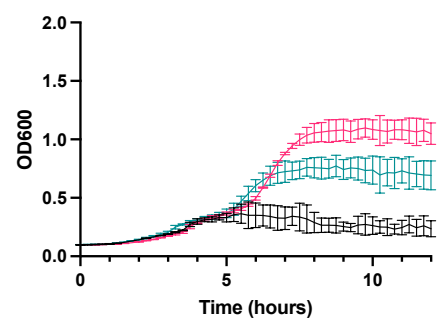

BREX

(tet)

MOI 0.01

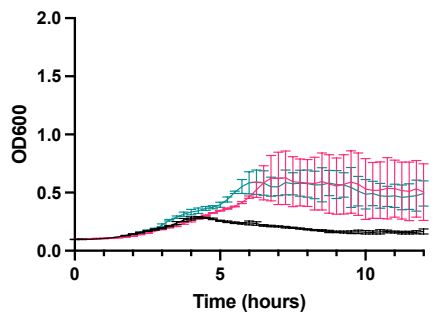

MOI 0.1

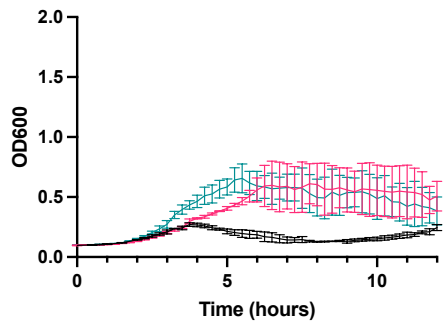

- PACYC Empty Vector

- WT-Brex-TET

- WT-Brex-5' Promoter 
b

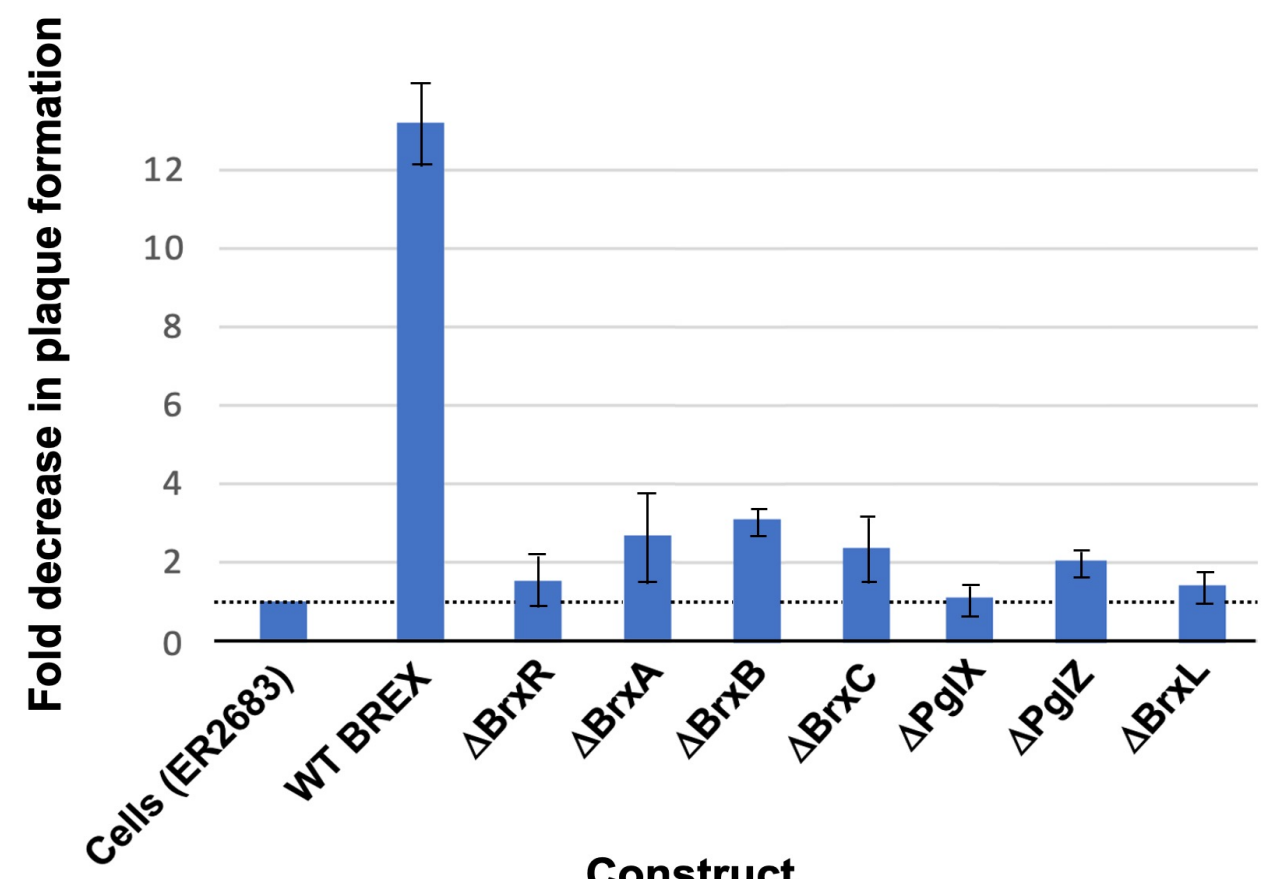

Cells (ER2683)

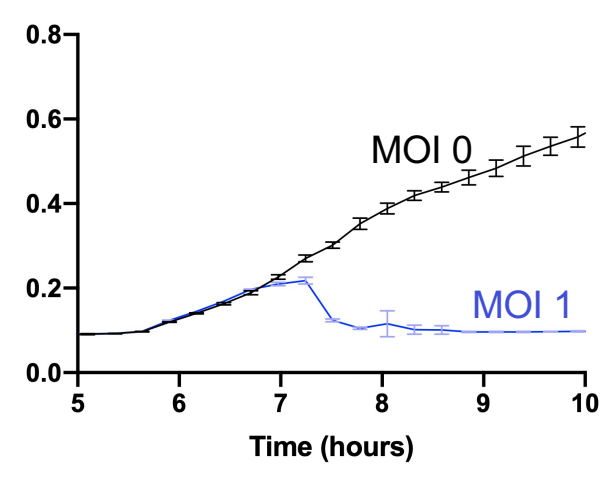

$\Delta \mathrm{BrxA}$

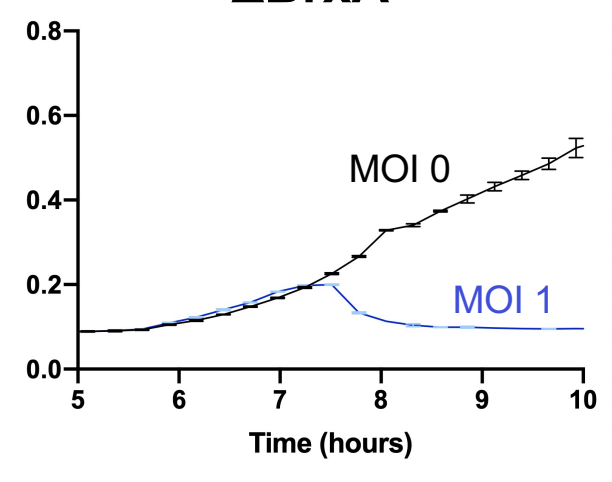

$\Delta \mathrm{PgIX}$

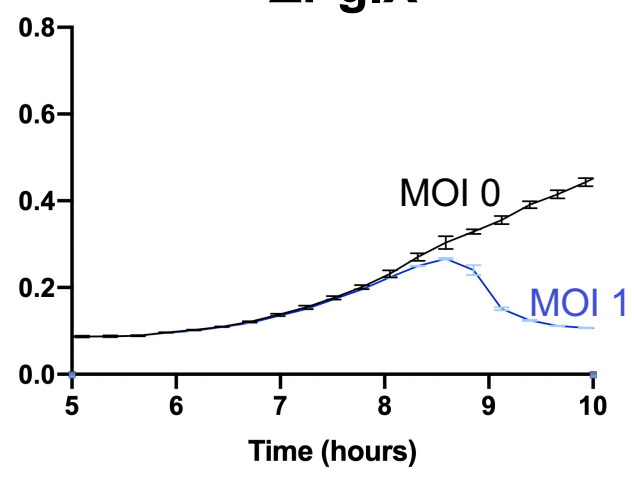

WT BREX

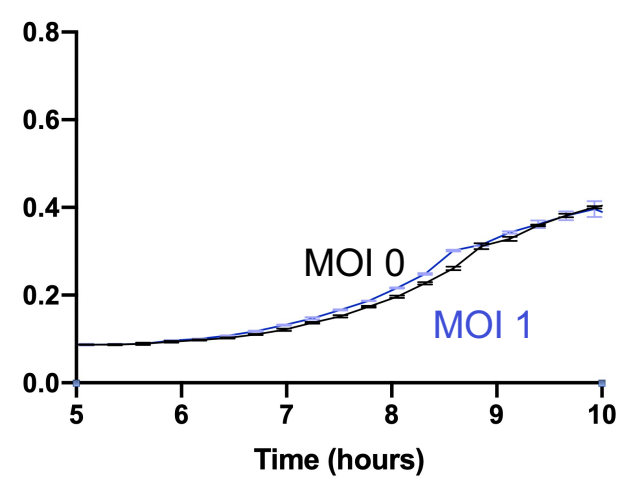

$\Delta \mathrm{BrxB}$

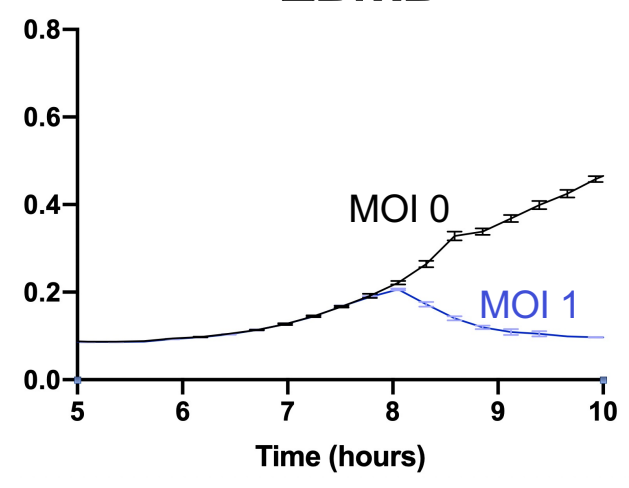

$\Delta \mathrm{PglZ}$

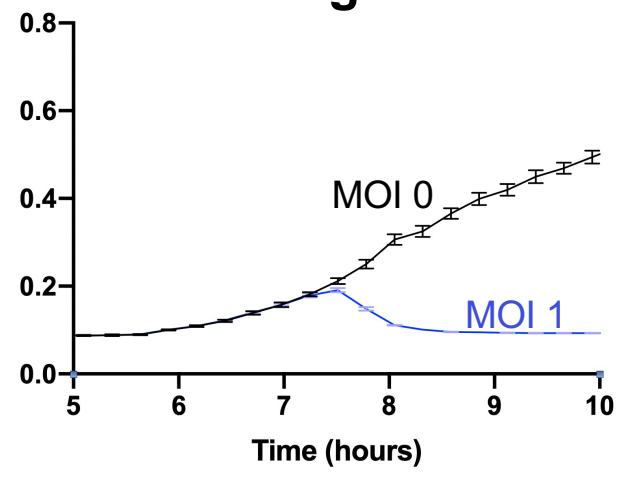

$\Delta \mathrm{BrxR}$

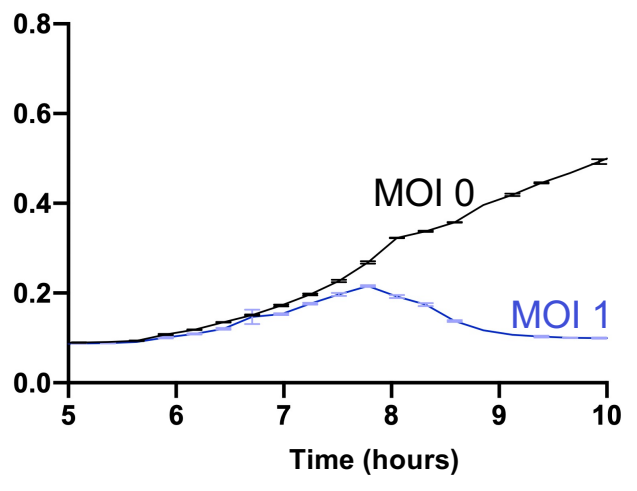

$\Delta$ BrxC

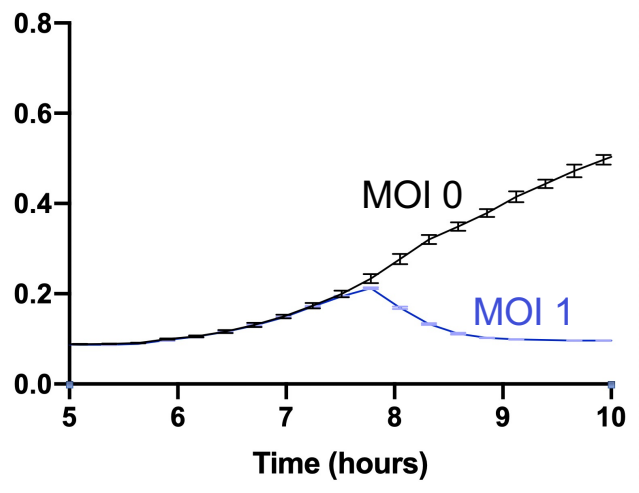

$\triangle \mathrm{BrxL}$

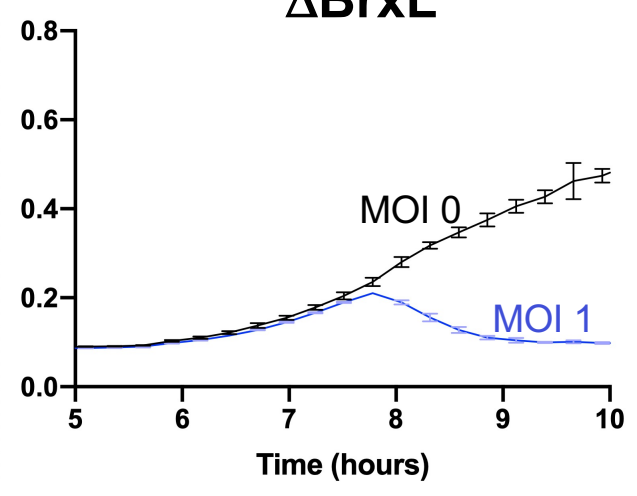




$\begin{array}{lccccc}1 & 71 & 119 & 197 & \\ & & & & & \\ \text { wHTH } & & \text { WYL } & & \text { WCX }\end{array}$
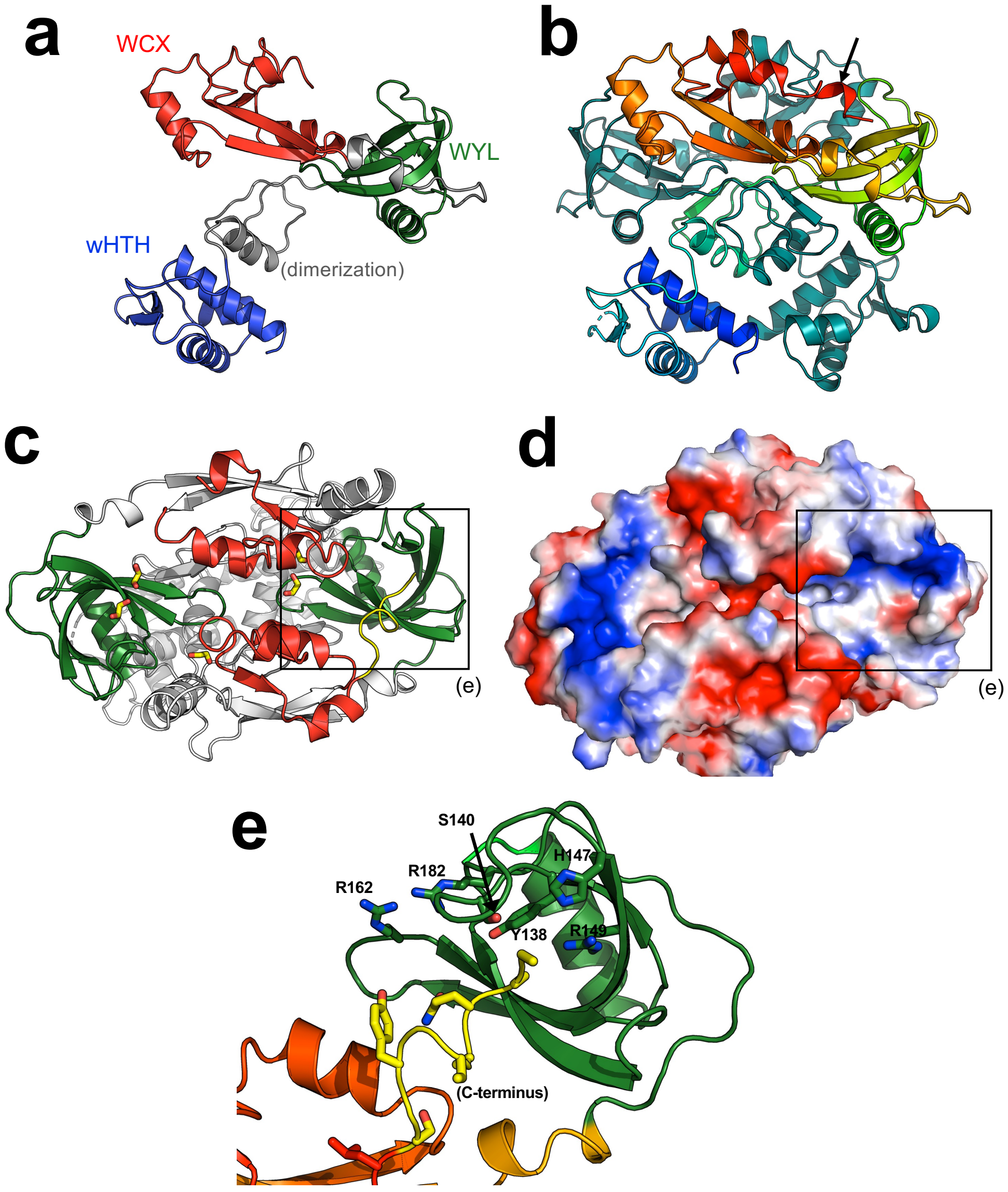
a

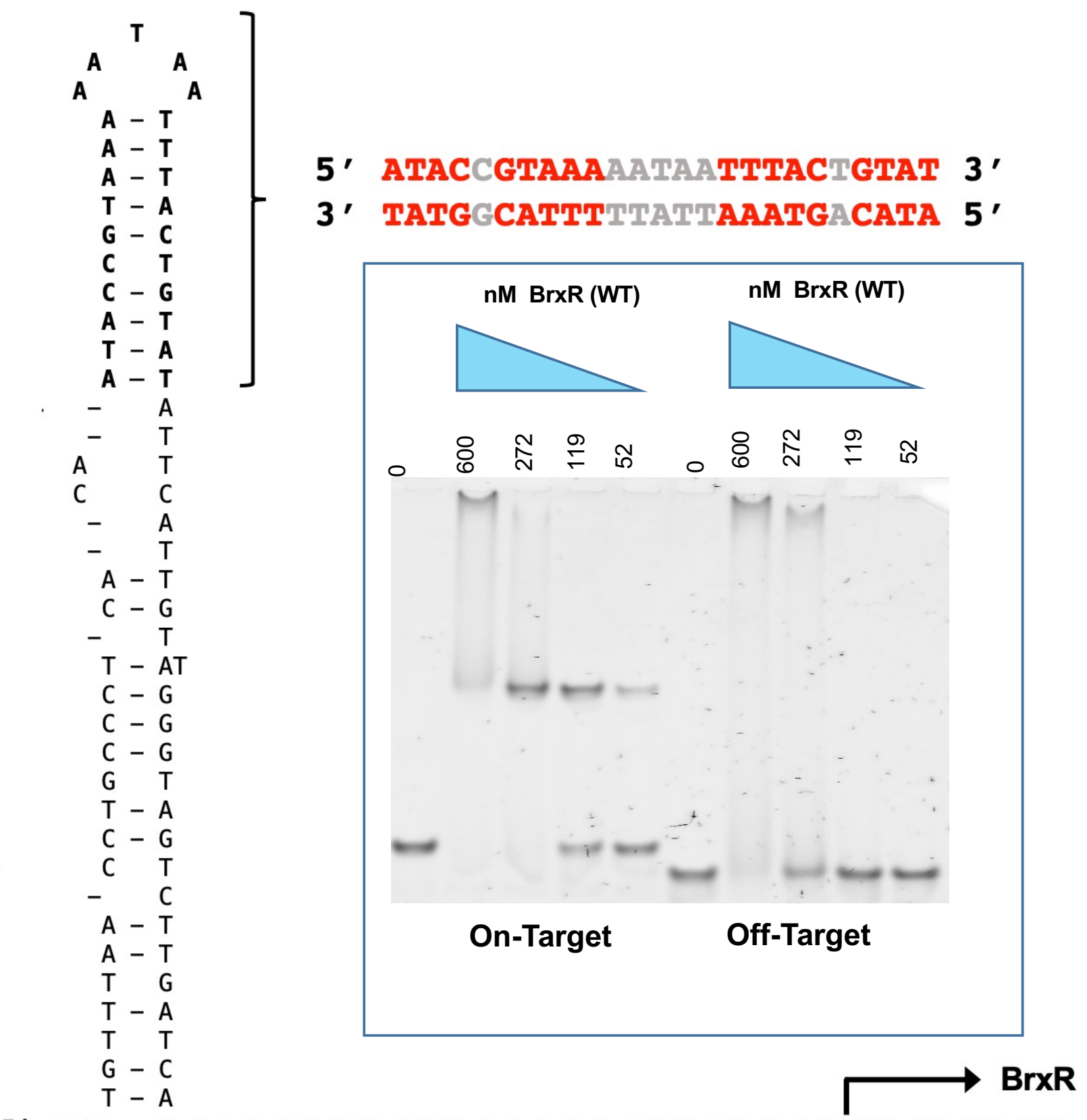

5'-gata C A T T G T T T A T T G A C C G A T T G T T C T ATG ACA GCA GAC AAG CAT. . .

BrxR binding site

cctcacaATACCGTAAAAATAATTTACTGTATattcattgtagggtagtcttgatcacATTGTTTATTGACCGATTGTTCTatg BrxR gene... 
bioRxiv preprint doi: https://doi.org/10.1101/2021.12.19.473356; this version posted December 19, 2021. The copyright holder for this preprint

a

(which was not certified by peer review) is the author/funder. All rights reserved. No reuse allowed without permission.
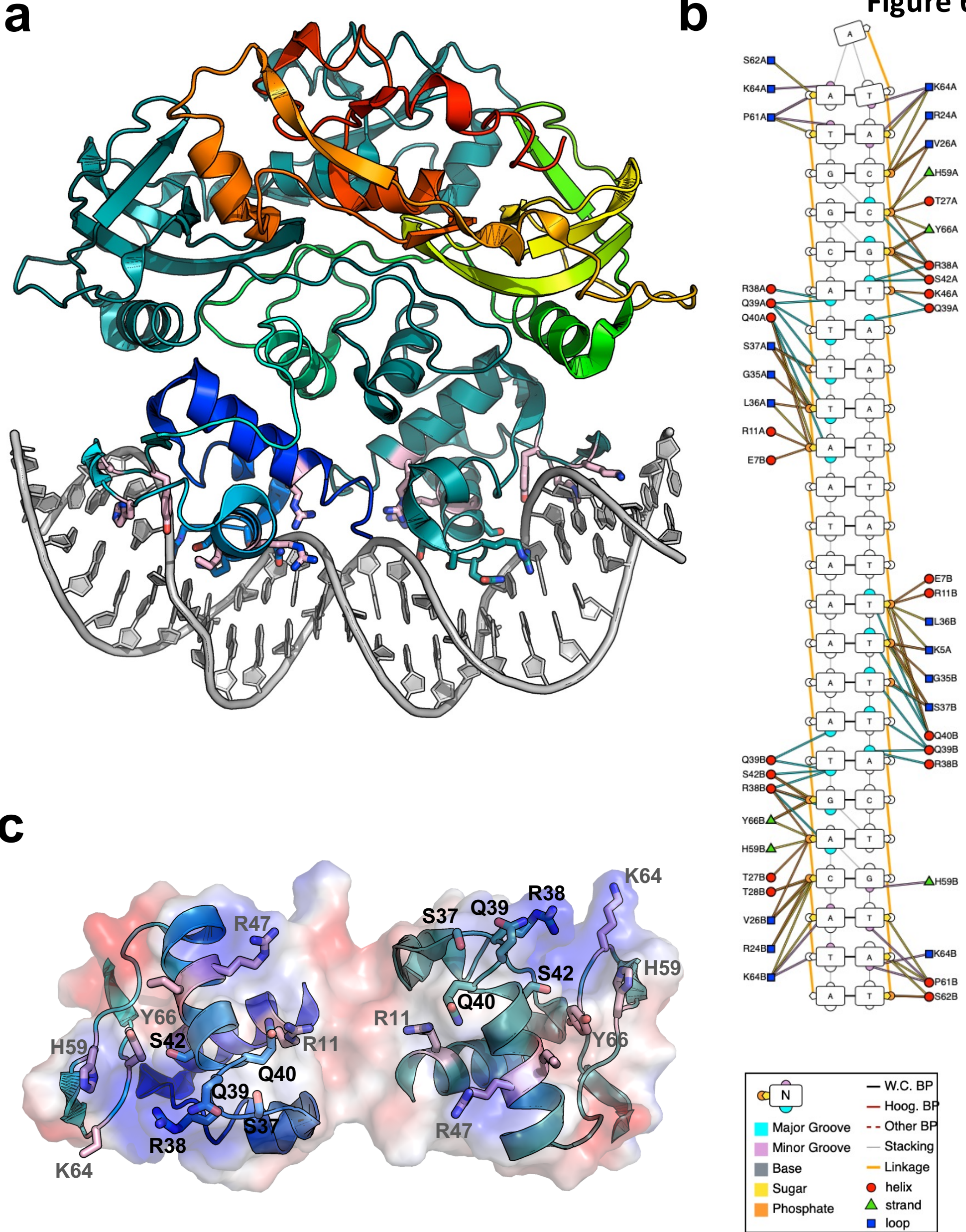


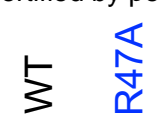
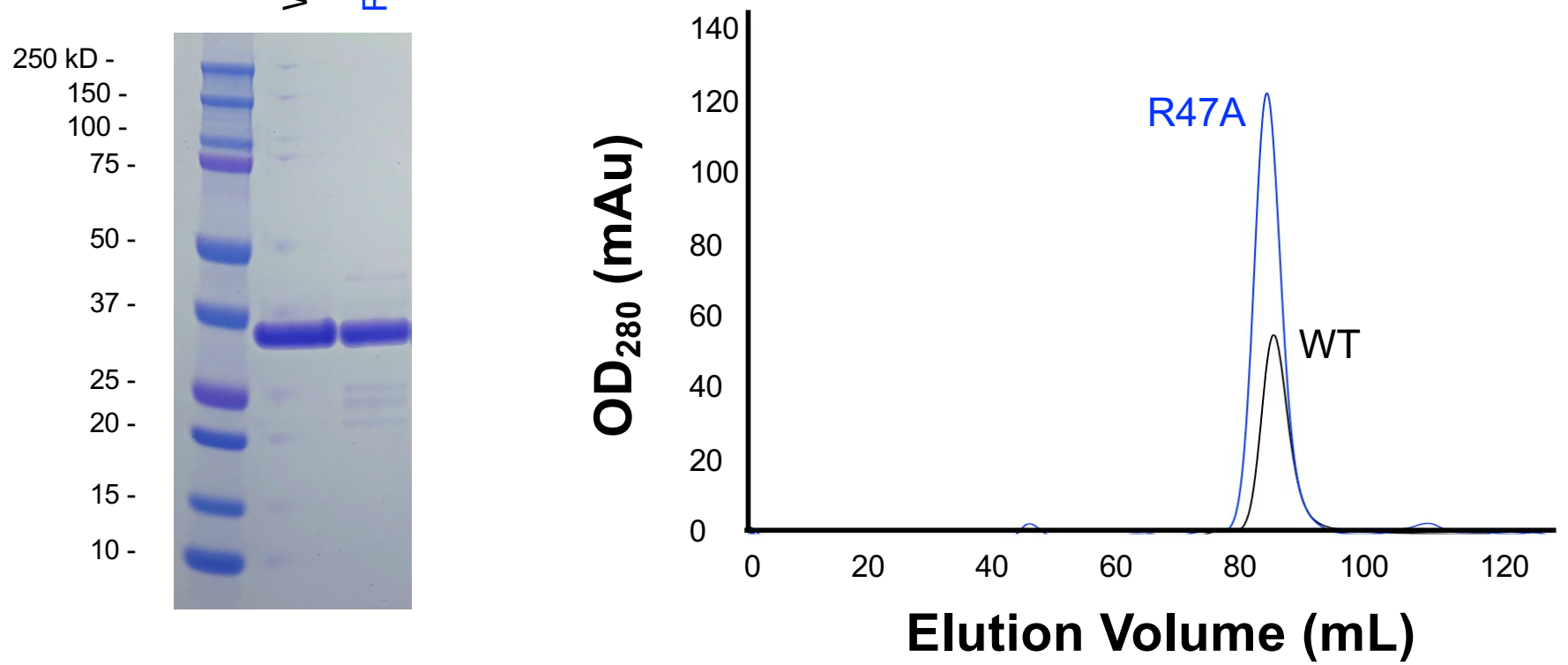

C

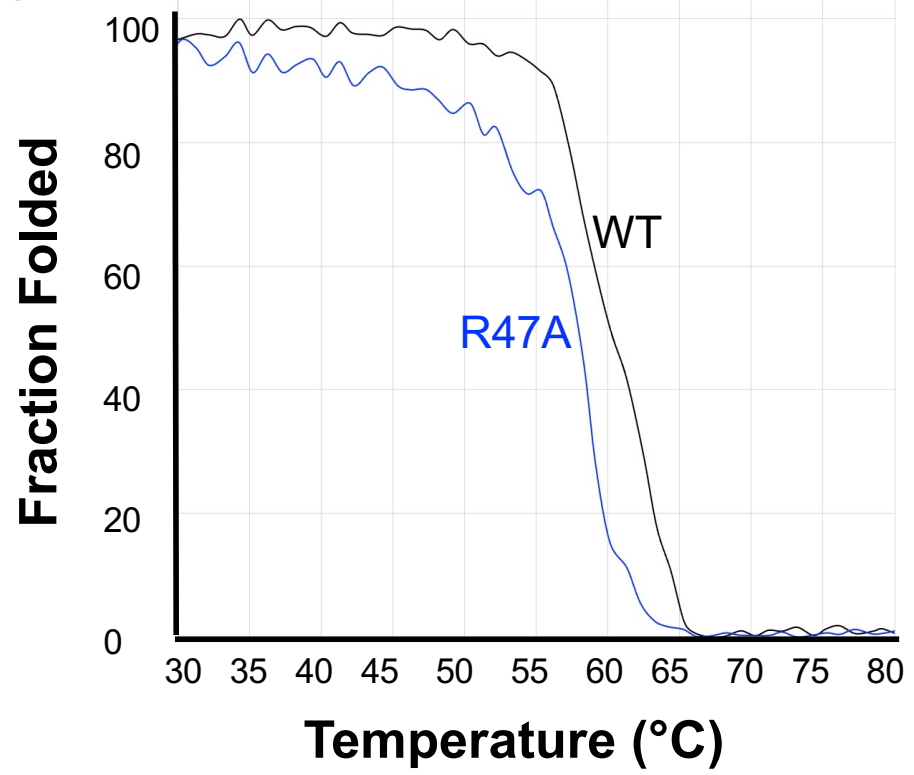

$\mathbf{e}$
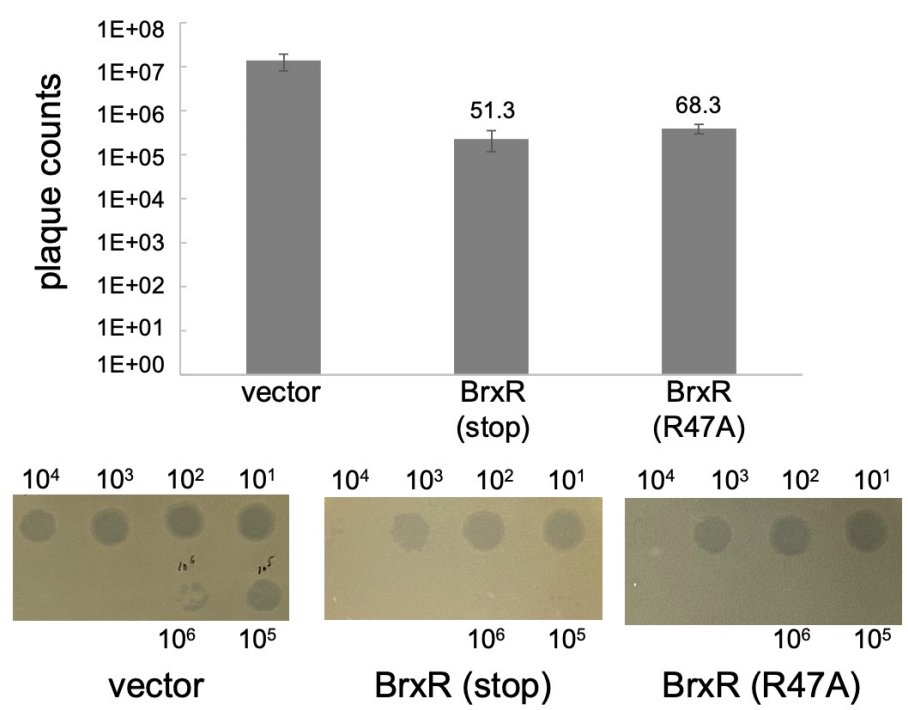

0
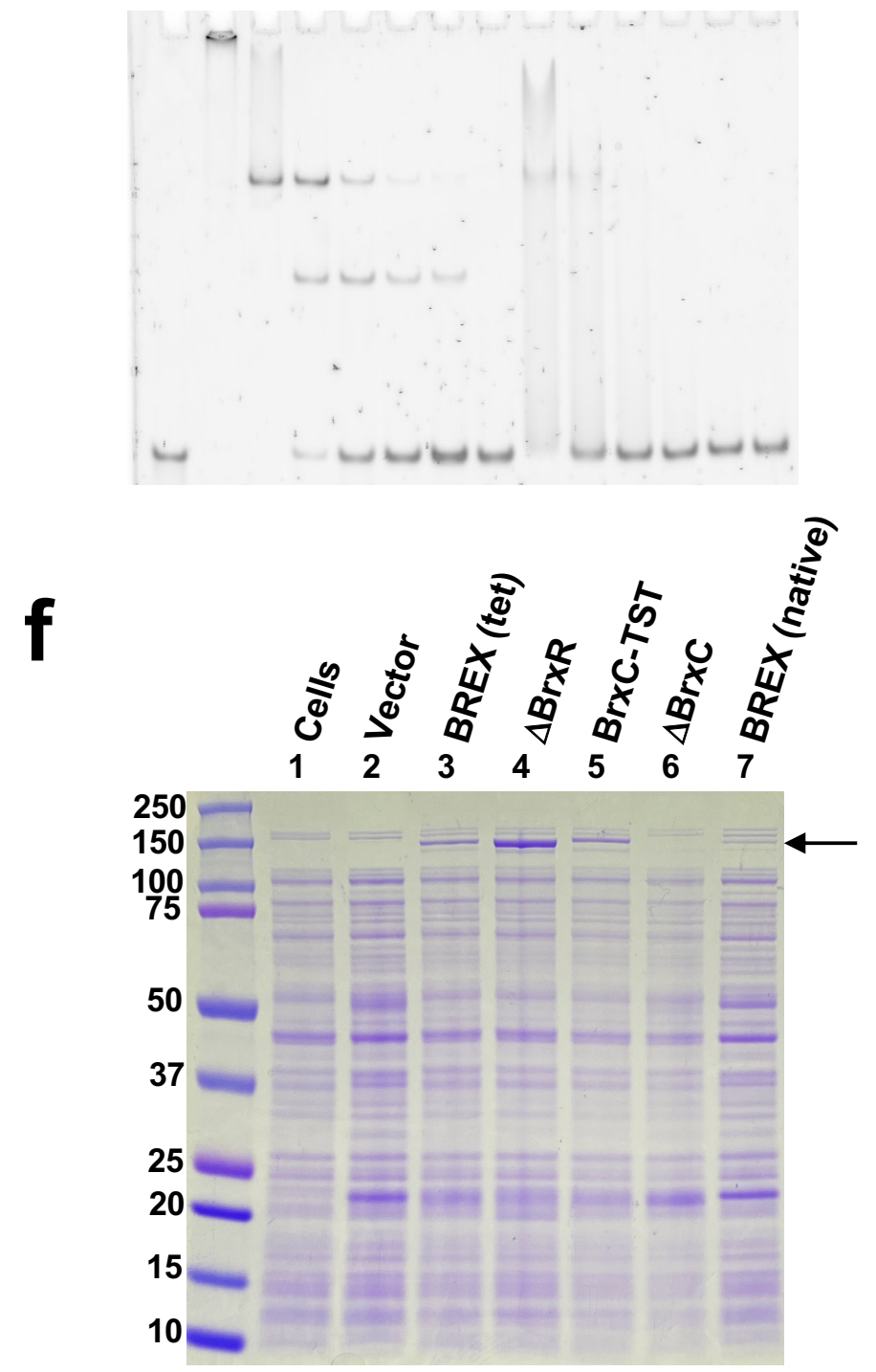

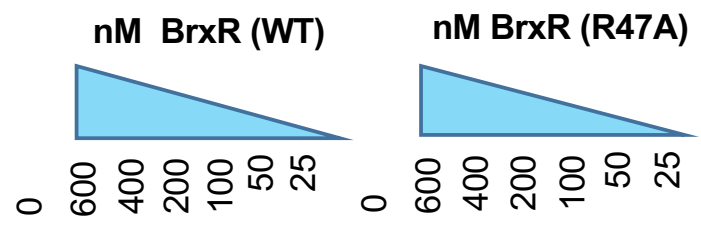




\section{SOURCE}

Acinetobacter Klebsiella pneumoniae Vibrio tubiashii Burkholderia sp. Thalassolituus marinus oceanospirillaceae Marinobacterium lutimarus Pseudomonas putida Azotobacter chroococcum Alcanivorax profundimarus Escherichia fergusonii

\begin{tabular}{lllllll|}
\hline & $\boldsymbol{\alpha 1}$ & & \multicolumn{2}{c|}{} & & \\
10 & 20 & 30 & 40 & 50
\end{tabular}
--------MTADKHEVLLRMRAIELLAYWEGRLVTTRLMNWFGLSRQQASADIKRYNT --------MTTDKYEVFLRMRAIELLAYWEGRLITTRLMNWFGLSRQQASADIKRYNT --------MADEKHEVLLRLRA IELLAYWEGRLVTNRLMSWFGISRQQASSDIKRYIS --------MSVLKHEALLRLRA IE LIAYWEGRL I TPQLMDWFG ISRQQASADIKYYNS --------MSTEKYEVLLRMRA IELLAYWEGRLVTNRLMSWF GISRQQASADIRRYVD -----MASVLADLKWEVLLRYRYIEI IAHWEGRLTTNHLCSTFGIKRQQASRDINQYK-----MSEPNEDLKWEVLLRYRYIEI IVLWEGRLTTNHLCNTFGIKRQQASRDINQYK----MKRKQS IAQVRWDLALRYRLIETIVWWEGRLTTGHLMQS FGISRQQASKDINTYIT ----MKREQS IAQVRWDLALRYRLIETVAWWEGRLTTQHLMQSFGISRQQASKDINTYIT MARVSRKSAGEWPVRWDLLLRYRLIEVVALWEGRLTTNHLMRAFGIGRQQASKDINTYLA -----QDNTGLEELSQAQRERLAHIDFTLLFKGEAGRSYLTERFSVAPSVATQDFARYKA Figure 8 IDENTITY
$100 \%$

$92 \%$

$69 \%$

$62 \%$

$53 \%$

$45 \%$

$43 \%$

$43 \%$

$40 \%$

$40 \%$

$22 \%$

\author{
Acinetobacter \\ Klebsiella pneumoniae \\ Vibrio tubiashii \\ Burkholderia sp. \\ Thalassolituus marinus \\ Oceanospirillaceae \\ Marinobacterium lutimarus \\ Pseudomonas putida \\ Azotobacter chroococcum \\ Alcanivorax profundimarus \\ Escherichia fergusonii
}

Acinetobacter Klebsiella pneumoniae Vibrio tubiashii Burkholderia sp. Thalassolituus marinus Oceanospirillaceae Marinobacterium lutimarus Pseudomonas putida Azotobacter chroococcum Alcanivorax profundimarus Escherichia fergusonii

Acinetobacter
Klebsiella pneumoniae
Vibrio tubiashii
Burkholderia sp.
Thalassolituus marinus
Oceanospirillaceae
Marinobacterium lutimarus
Pseudomonas putida
Azotobacter chroococcum
Alcanivorax profundimarus
Escherichia fergusonii

Acinetobacter Klebsiella pneumoniae Vibrio tubiashii Burkholderia sp. Thalassolituus marinus oceanospirillaceae Marinobacterium lutimarus Pseudomonas putida Azotobacter chroococcum Alcanivorax profundimarus Escherichia fergusonii

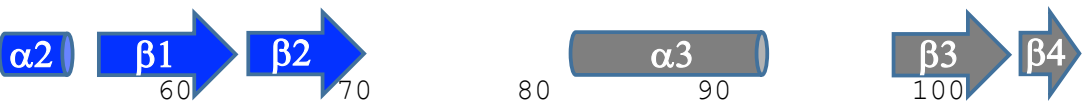

LYNPDALIHDPSVKGYVPKASFOPVLTTAHINEYLNMLSGLVSES--HALIAMPEPNLAA LYNPDALFHDPSVKGYVPKASFQPVLTTTHINEYLNMLSGLVSES--HALIATPEAHLSA VHNPGSLYHDPGVKGYVPQGDF $Q P V L T K G H I N E Y L D M I S G L V S E S--V A V T L E S E D H L A A$ KFNPDSLVHSPAVKGYVPTAGFQPVLSSGHINEYMDLLAGQGSQP--MAQVLENHSSVAT SYNPGALVHSPAVKSYIPSDTFTPILTTGHINEYLDLLVAFGDRS--ESSVEQSESMFVS ELAPANLLYDTKLKGYAPSPGFKPIFTAGSVDEYLNLLDSHSLLEGFIERVELPSPNTQV TLAPDNLAYDTSLKGYTPAPSFRPIFTTGYVDEYLNLLDSHSLLDGFVQRIELPSANTFV EHASRNLTYDKQLKGYVPSKTFNPRFIDLSASAYLDLLAQNNERAPHIEGLKLAYAHTLV EHAPKNLTYDRHLKGYVPAAKFKPLFIDDSADAYLHLLNQSHERAPHIEGLALAYAHTEV DYGPDNLVYDRELKGYRPTPGFRPVFTRGVADEYLQMMHAREDLASSEEALTVRELNTEV L-APNNVMYDEKRRVHLKTSTFQPLFDYDIVRTLATISQGFGDGFLGKVRPPMACEAPFH

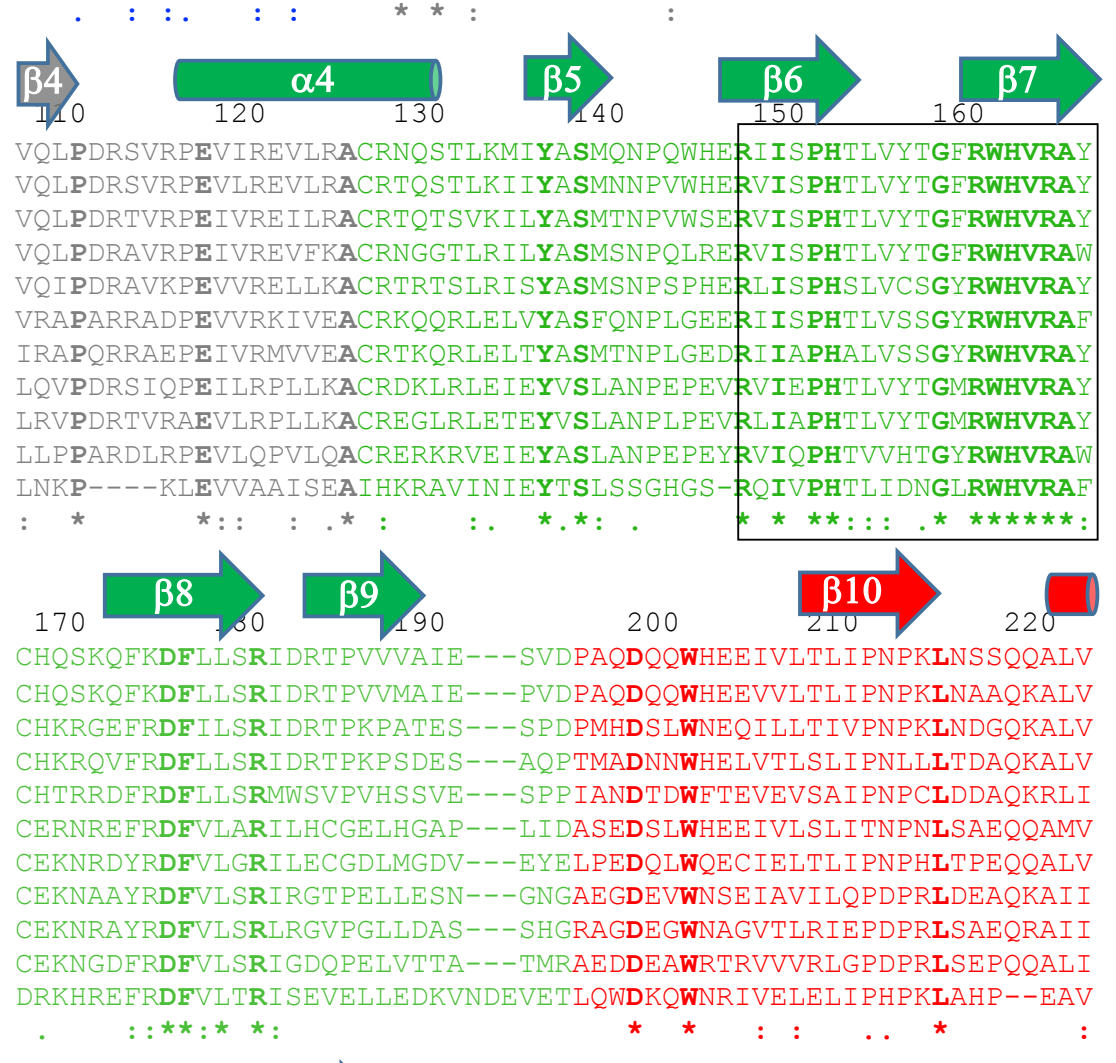

a5

$\beta 11 \quad \alpha 6$

$\begin{array}{llll}240 & 260 & 270 & 280\end{array}$ EKDFGMPDGRLQI PVKKALAHYTLQRYQTAIT---LAEAEDALKYPLVLQRSDIEKLSSYLFDQAS--- - EKDFGMPDGRLQI PVKKALAHYTLQRYQAAIT---LAEAEDALKYPLVLQRSDIEKLSSYLFDQTS-----EKDFGMPDGRLQISVRKALAHYTLQRYQAAIT---VDEAADEFKFPI I LLDSDRKKLSPFLFGSES-----ERDFGMPEGHLQLTVRKALAHYTLQRYQAAIT---EQEAERLREHPLQLLPGDRAKLEPYLLGGEDE----EMDYCMTDGVLRIRSKIALLHYTLQRYQIGVR---NEDRADAKSFPI I LDNDSRCKVDKSLFCGAAFD---RLERCFDDEVMQIPTRRALAIYLLQLMQVPTEPLHEDERKNPAAFPVVLDDYHQLQELSFGRKIG------RHERCFNGPTLTLETRKATAIYMLQLLQVPTSAPAS DSPDNVNANPLVLEDYKLIEELKFEQTRAGRANTRH QRDYGMIDGRLEIPTRQALVKYVLQRYQIDPK---NLDPKAEAQQVVIGN---LSELKPWLYD--------ETDYGMRDGHLAVASRGALVQYVLQRFQIDTT---KIEPKAAAQQIVVAN---LEELARWLYK--------QRDYGMEQGELRLETRGALVSYLLQQLRVDAP---ADQRS PEAQQVVVLN---RDEVEQWALMPG------LIDYAMENNRLRVEIRAAFAGYLLRLWNIDCS---KNSKSNGREFHLALKNPEALYGVDNAALAPGYSES-- 


\section{SUPPLEMENTARY FIGURES}

Supplementary Figure S1. E. coli ER2683 cells transformed with empty vector, vector encoding the WT BREX operon, or the same operon harboring precise deletions of each BREX protein factor. Reduced colony size and transformation efficiency is observed for $\Delta \mathrm{BrxL}, \Delta \mathrm{BrxB}$ and $\Delta \mathrm{PglZ}$.

Supplementary Figure S2. Combination of PacBio SMRT capable seq and Illumina capable seq TSS data. Panel a: PacBio SMRT capable seq data for the full-length BREX operon. The top track shows BREX reading frames. The next two tracks show Illumina read capable seq pile ups (read counts) from two independent experiments. The bottom panel is PacBio SMRT capable seq showing coverage (gray) and then mapped reads (salmon). This shows a strong TSS just before BrxR and the BREX operon, with only very low amounts of internal operon reads, indicating one TSS for the entire operon. Panel b: SMRT capable seq with a detailed view of the region upstream of the BrxR reading frame and the corresponding transcription start site. Panel c: Sequence of the Acinetobacter 394 BREX 5' UTR, indicating the location of the BrxR binding site, predicted promoter region, transcription start site (grey arrow) and the start codon for the BrxR gene ('atg' and black arrow).

Supplementary Figure S3. Phage restriction in E. coli NEB-5 $\alpha$ by BREX. Panel a: Growth of E. coli strain NEB5 $\alpha$ (New England Biolabs) transformed with pACYC184-BREX and challenged with $\lambda_{\text {vir }}$ phage at MOl's ranging from 0.1 to 0.001 . BREX' cells display lysis within five hours of the challenge, versus continued growth and saturation of the cells that harbor the BREX system. The level of protection conferred by the intact BREX system displayed a dependence on the phage MOI; cells containing BREX eventually crashed at the highest $\mathrm{MOI}(0.1)$ but continued to grow at MOI's of 0.01 and 0.001 .

Supplementary Figure S4. E. coli strain ER2683 transformed with pACYC-BREX constructs containing the indicated single deletions were challenged with $\lambda_{\text {vir }}$ phage at an MOI of 0.01. All BREX ORF's were required for restriction.

Supplementary Figure S5. Electrophoretic mobility ('gel shift') analyses of BrxR interaction with 13 regions distributed across the BREX operon (positions indicated in top schematic). For each experiment (which are distributed across multiple gels in a composite figure below) lanes with a constant $10 \mathrm{nM}$ concentration of dsDNA probe are incubased with $0 \mathrm{nM} \mathrm{Brx0}$ (left-most lane) and then a decreasing titration series of BrxR concentrations corresponding to 800, 400, 200 and $100 \mathrm{nM}$ BrxR. In all cases, no observable specific binding is observed at $100 \mathrm{nM} \mathrm{BrxR}$, and increased concentrations produce a nonspecific smear of shifted DNA species. 
Supplementary Figure S6. Overlay of the apo- (cyan) and DNA-bound forms of BrxR shows that the protein undergoes very minor conformational changes upon binding DNA. The two forms share an RMSD across all $\alpha$-carbons of approximately $0.8 \AA$.

Supplementary Figure S7. Purification and characterization of BrxR point mutants. A series of six point mutations (R47A, which is located in the protein's DNA binding domain, and five additional constructs located in the WYL domain) were purified (panel a) and demonstrated to all elute at volumes corresponding to dimers on a size exclusion column (panel $\boldsymbol{b}$ ). Subsequent determination of their thermal denaturation temperatures and unfolding behavior using CD spectroscopy demonstrated that one point mutant (R182A) was significantly destabilized (panels $\boldsymbol{c}$-d). One point mutant in the WYL domain (R149A) was analyzed for DNA target binding and shown to interact with that site in a manner similar to the wild-type protein (panel e).

\section{Supplementary Figure S8. WYL proteins upstream of defense islands in V. cholera are BrxR}

homologs. Vchlnd5, which was annotated by Legault et al as a "WYL" protein, shares 25\% amino acid sequence identify with BrxR. The predicted Alpha-fold structure of Vchlnd5 (left) closely matches the experimentally determined structure of BrxR, demonstrating that the $\mathrm{V}$. cholera WYL proteins are BrxR homologs. 

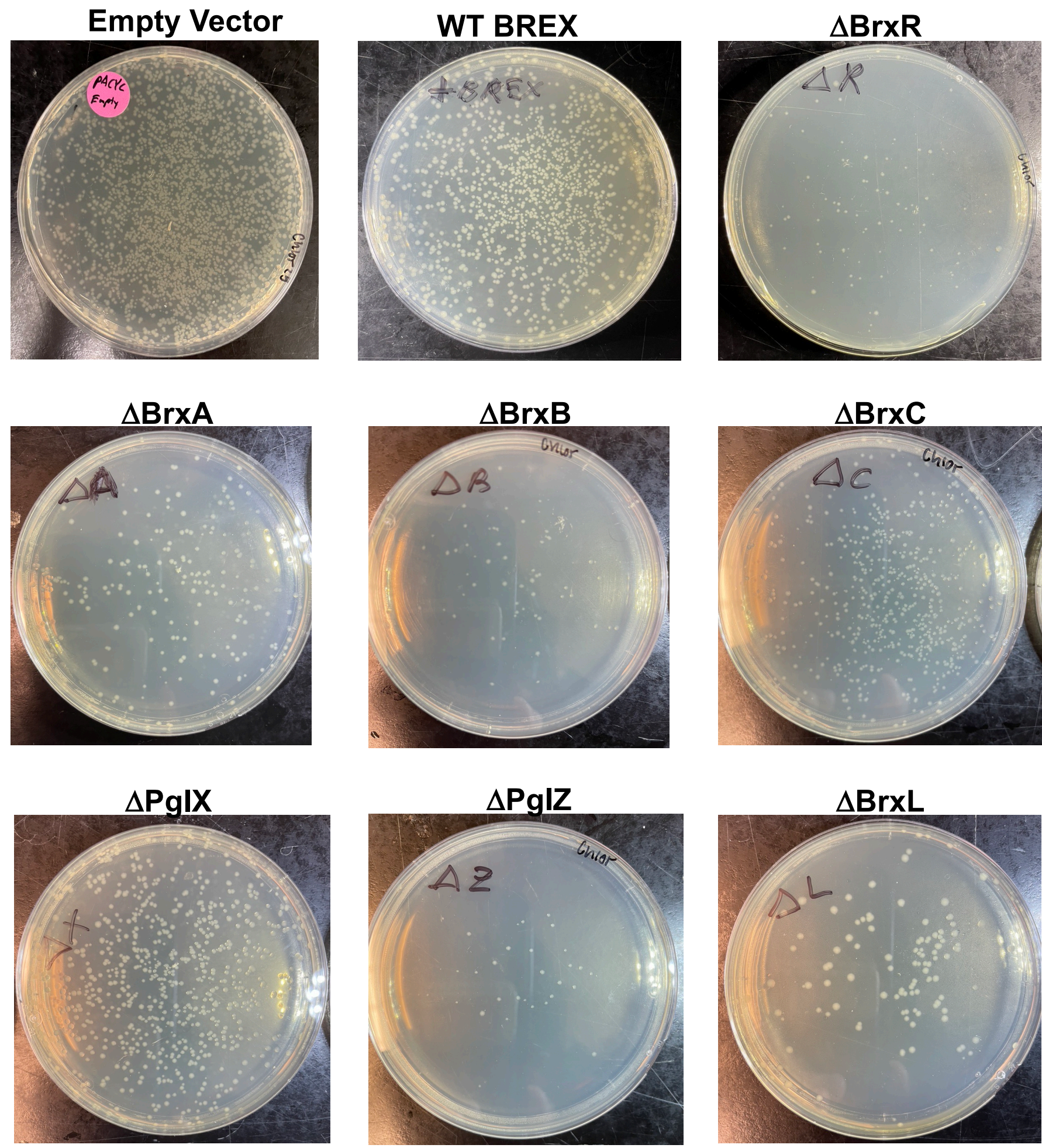
a

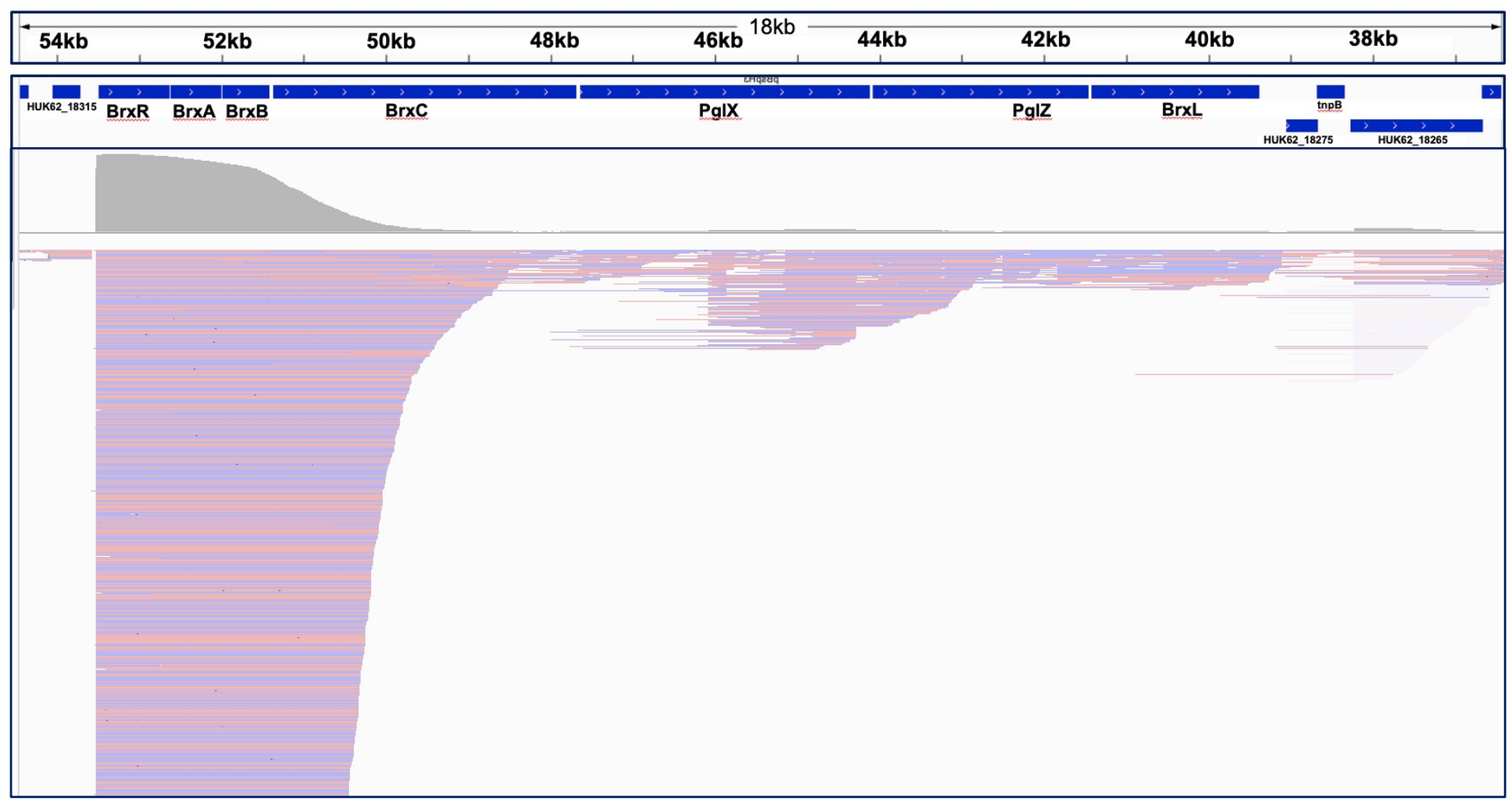

b

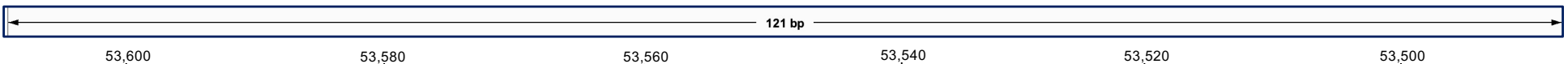

TTTGATCAGATATGTTTAACCTGCCCTCACAATACCGTAAAAATAATTTACTGTATATTCATTGTAGGGTAGTCTTGATCACATTGTT†ATTGACCGATTGTTCTATGACAGCAGACAAG Met-Thr-Ala-Asp-Lys

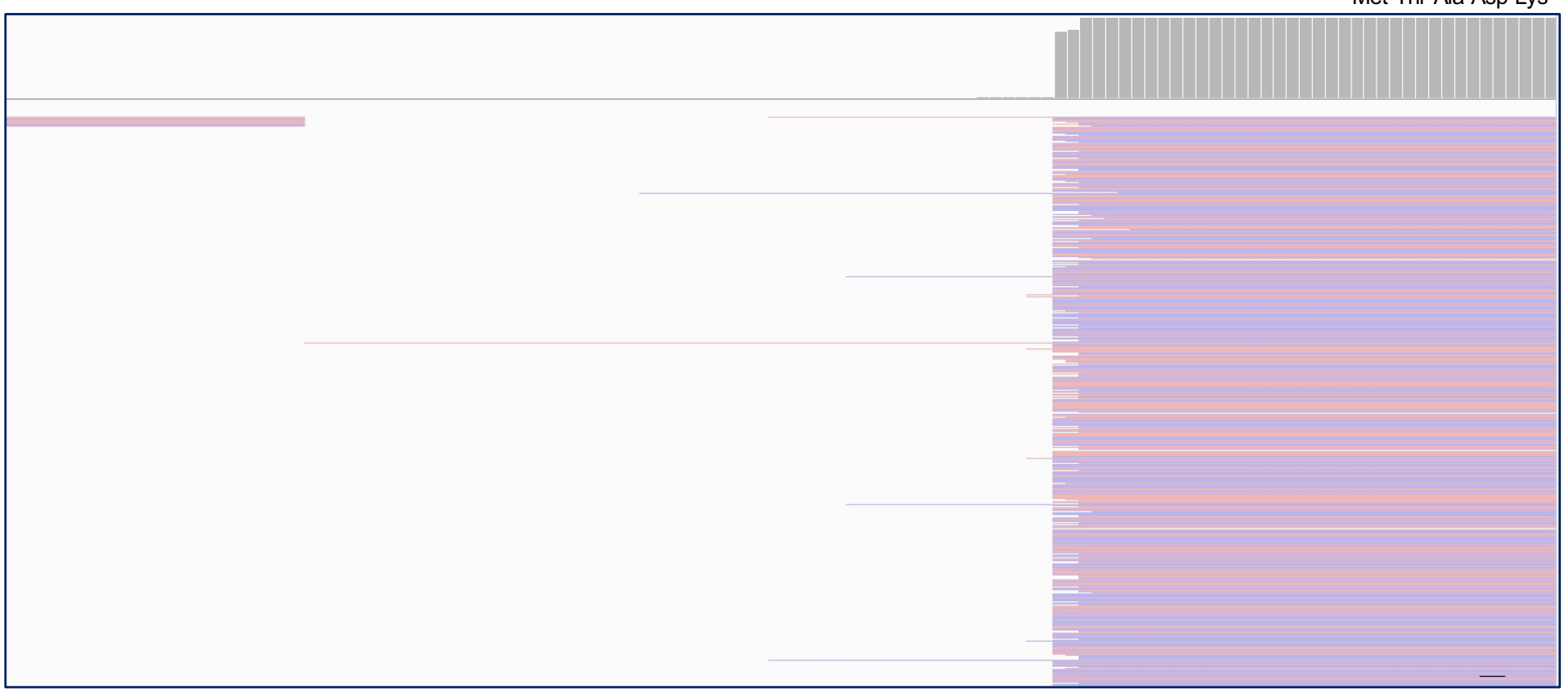

BrxR binding site

cctcacaATACCGTAAAAATAATTTACTGTATattcattgtagggtagtcttgatcacATTGTTTATTGACCGATTGTTCTatg BrxR gene... 
MOI $0.1 \quad$ MOI 0.01

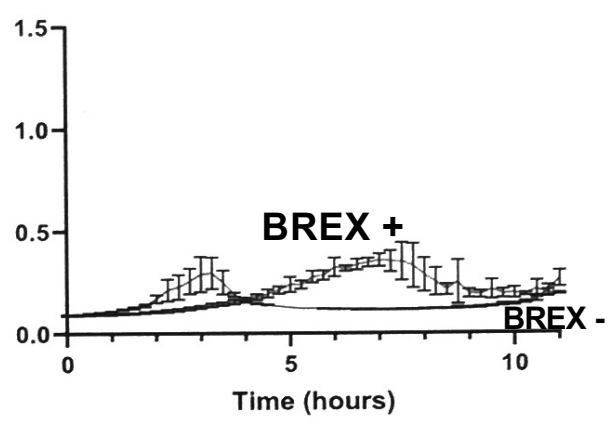

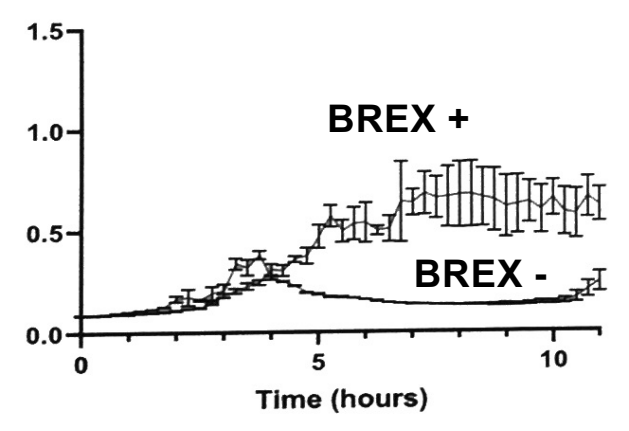

MOI 0.001

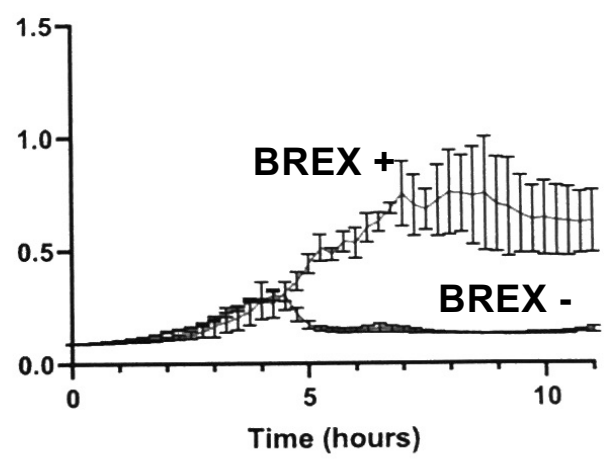



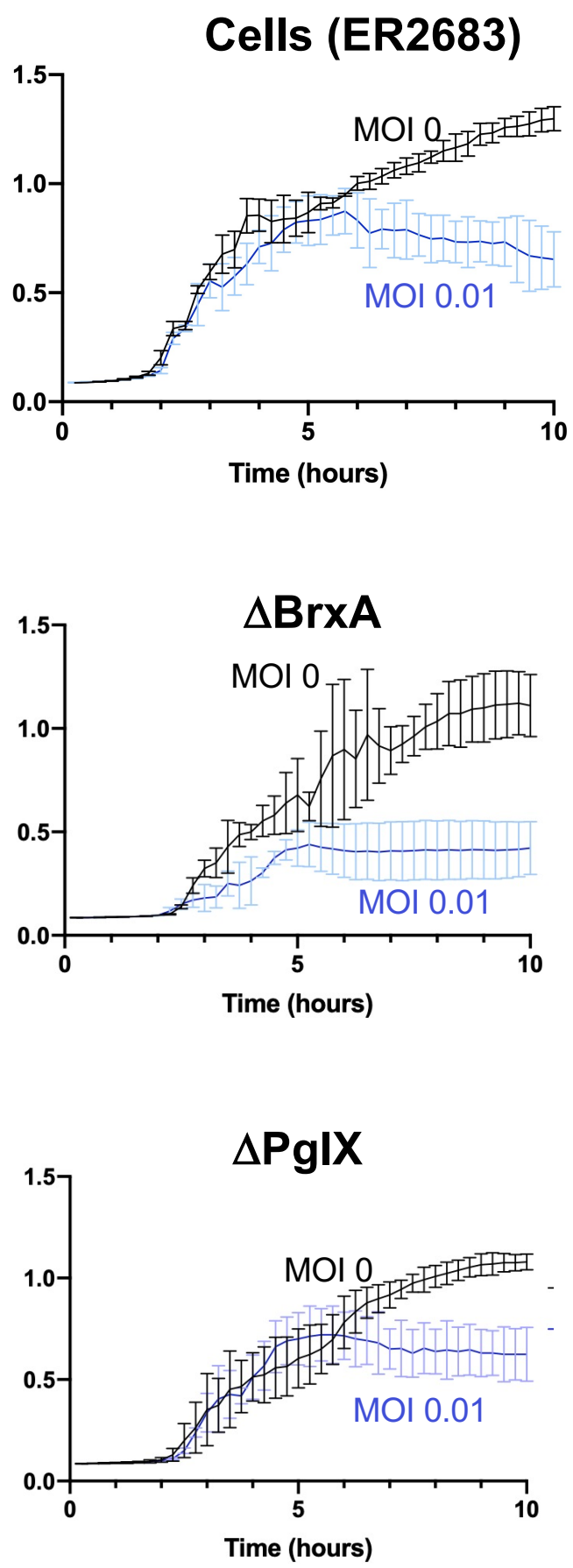

WT BREX
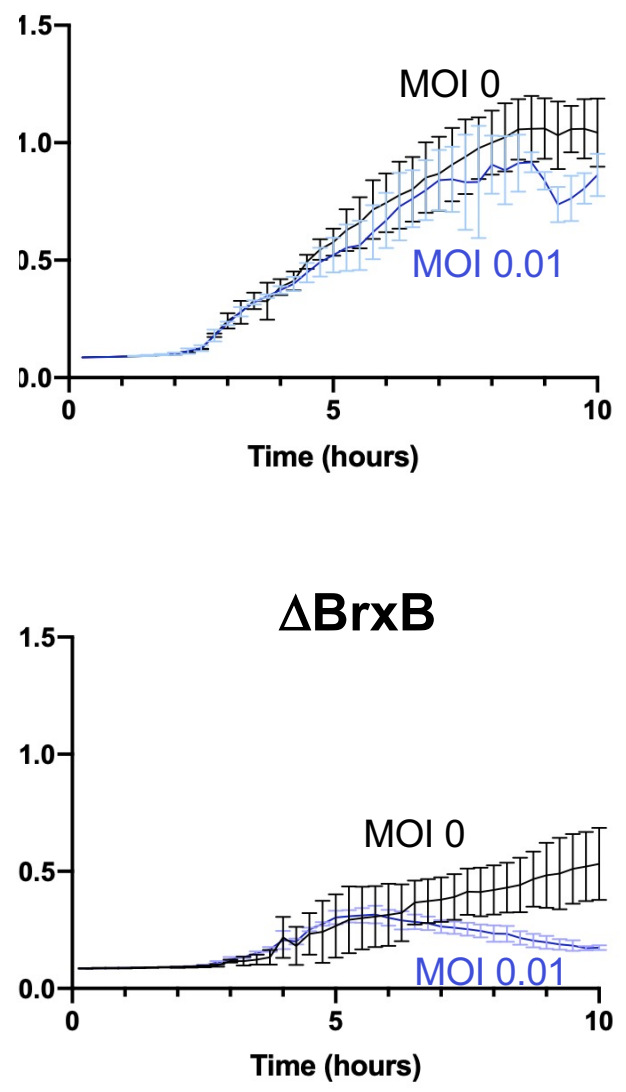

$\Delta \mathrm{PglZ}$

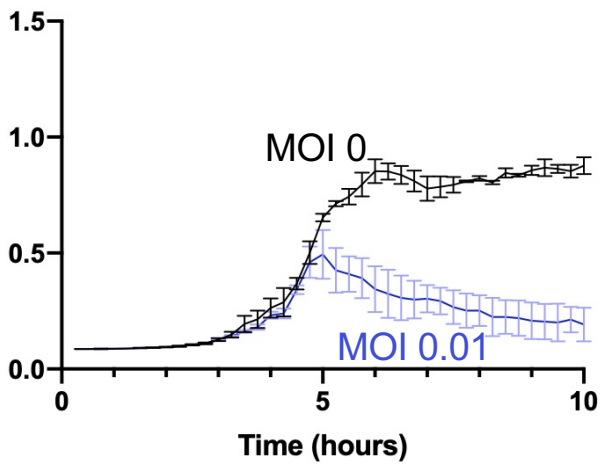

$\Delta B r x R$

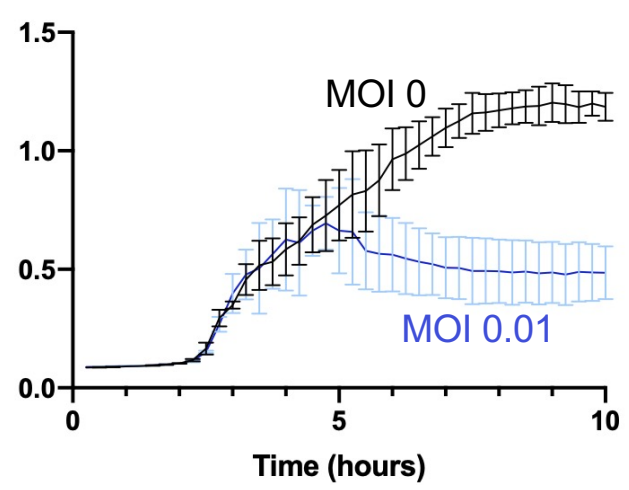

$\Delta B r x C$

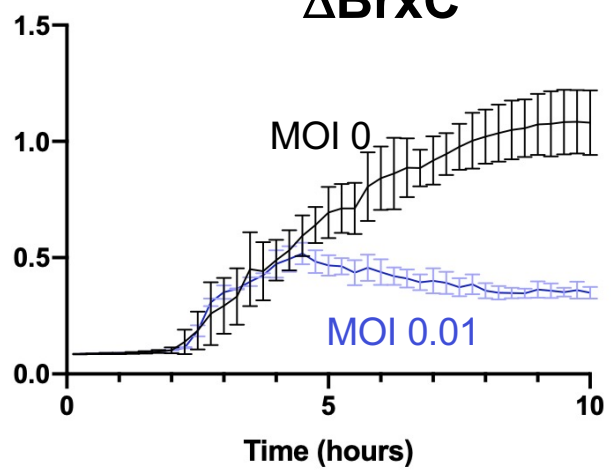

$\Delta B r x L$

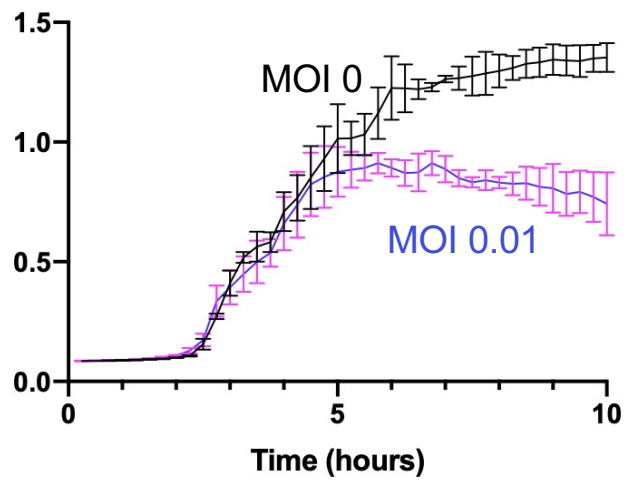



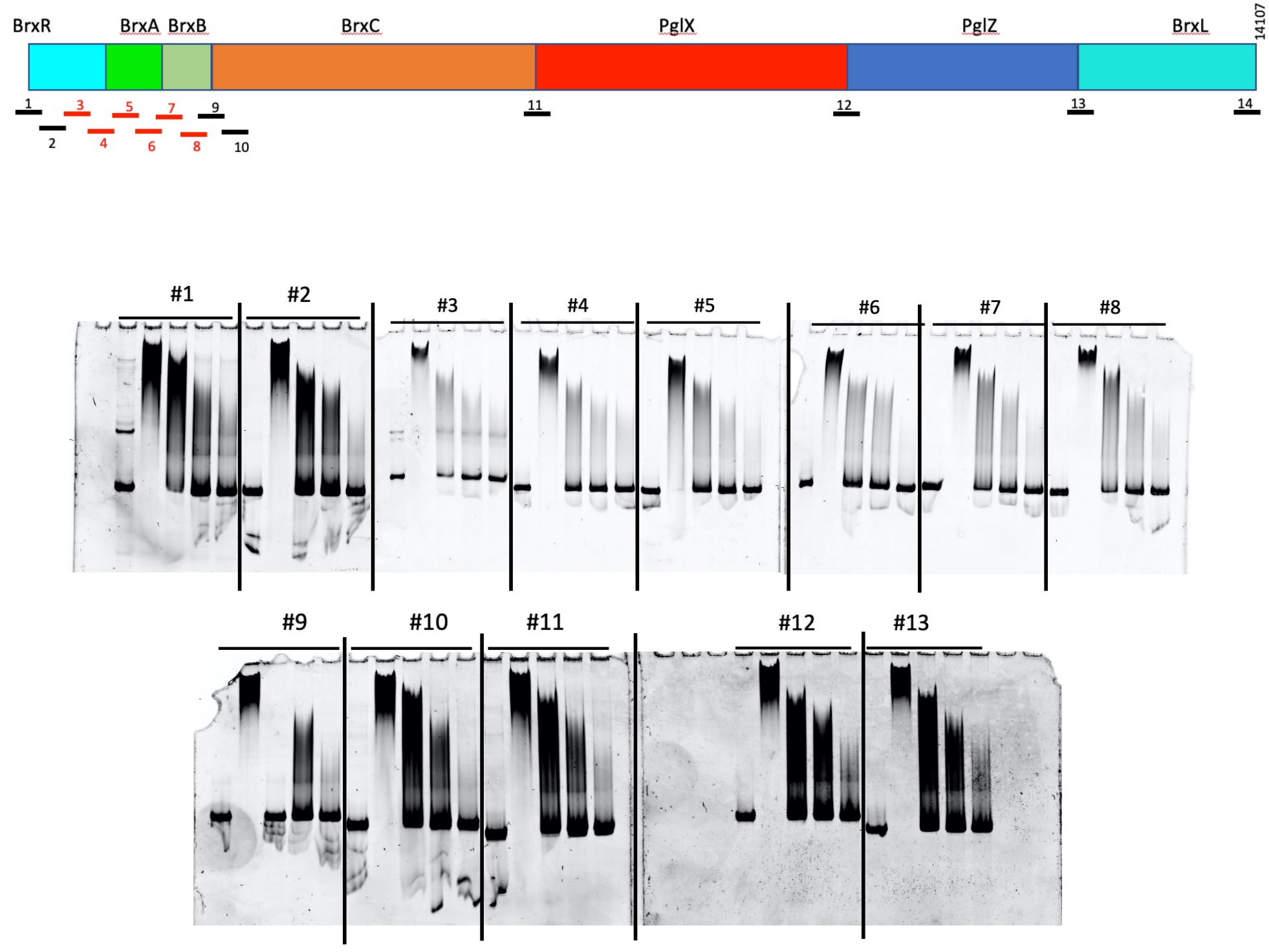


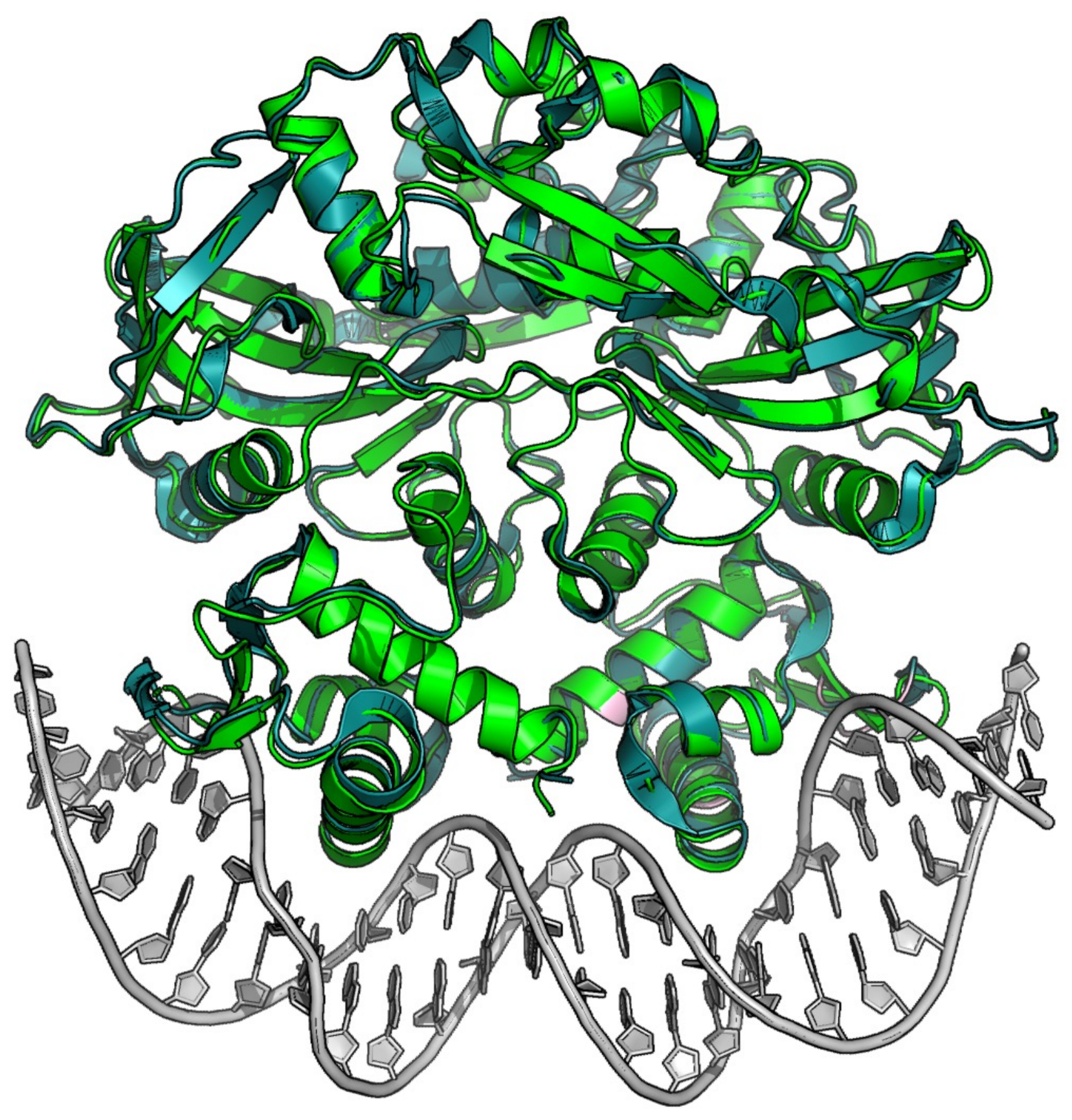


a

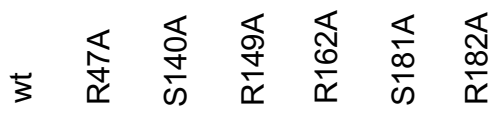

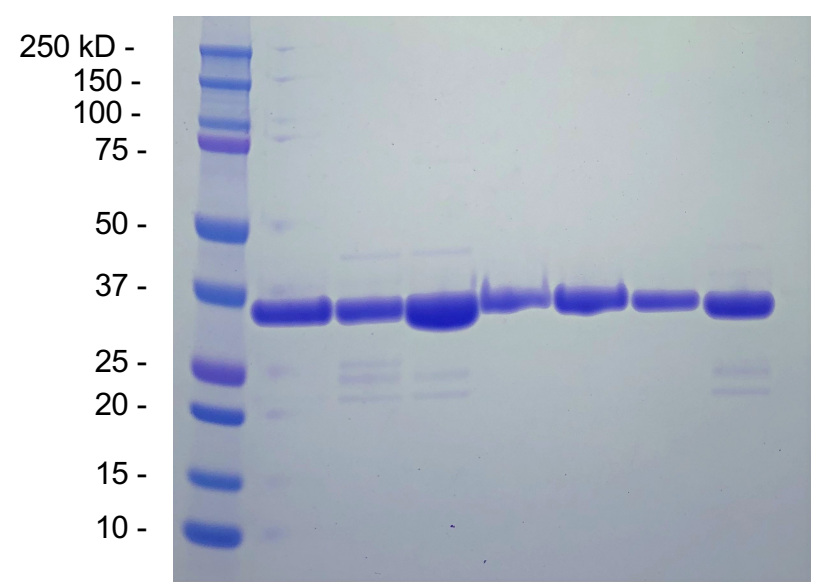

b
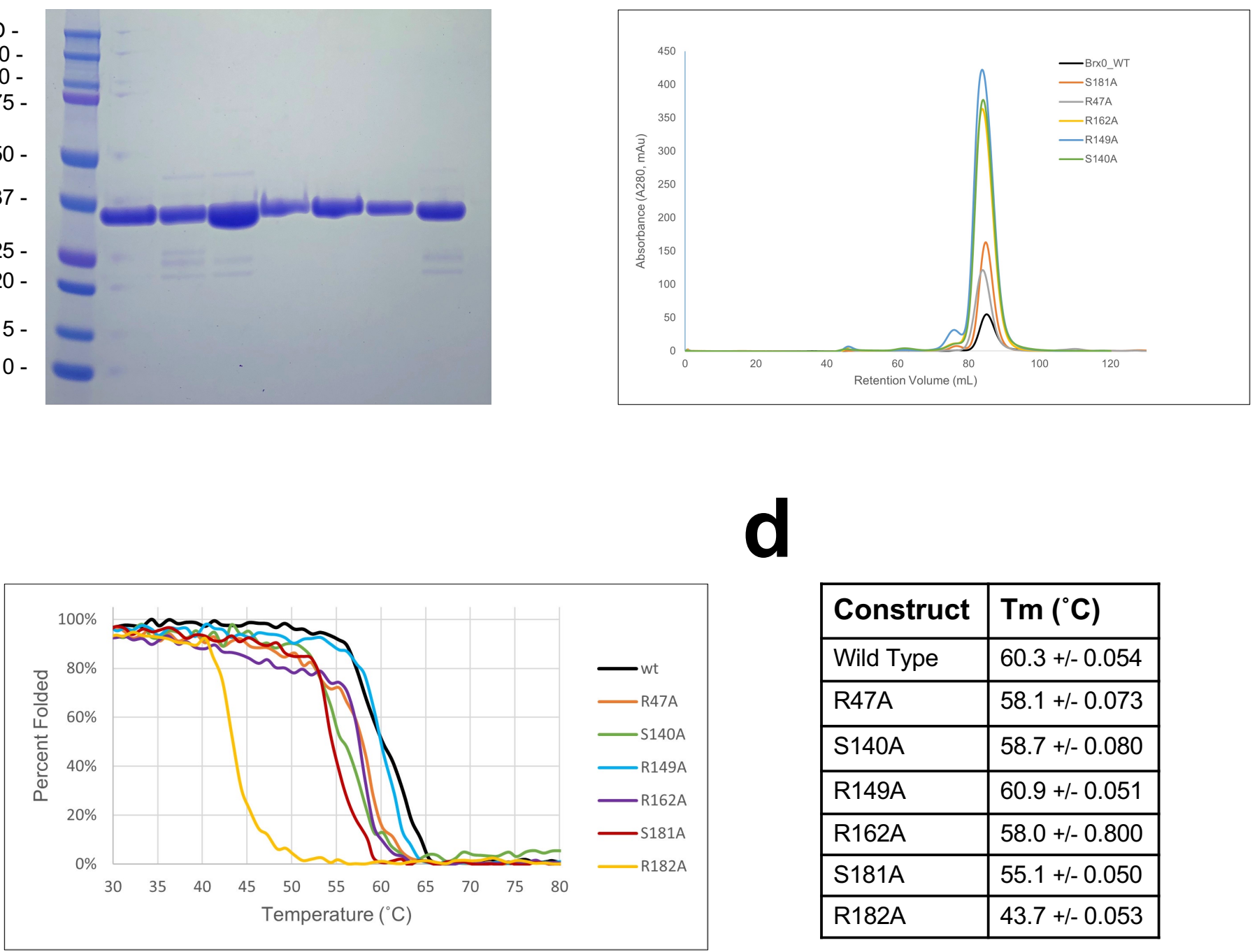

\begin{tabular}{|l|l|}
\hline Construct & $\mathbf{T m}\left({ }^{\circ} \mathbf{C}\right)$ \\
\hline Wild Type & $60.3+/-0.054$ \\
\hline R47A & $58.1+/-0.073$ \\
\hline S140A & $58.7+/-0.080$ \\
\hline R149A & $60.9+/-0.051$ \\
\hline R162A & $58.0+/-0.800$ \\
\hline S181A & $55.1+/-0.050$ \\
\hline R182A & $43.7+/-0.053$ \\
\hline
\end{tabular}

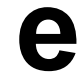

WT

R149A

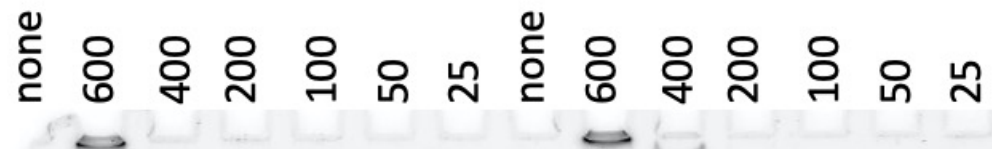




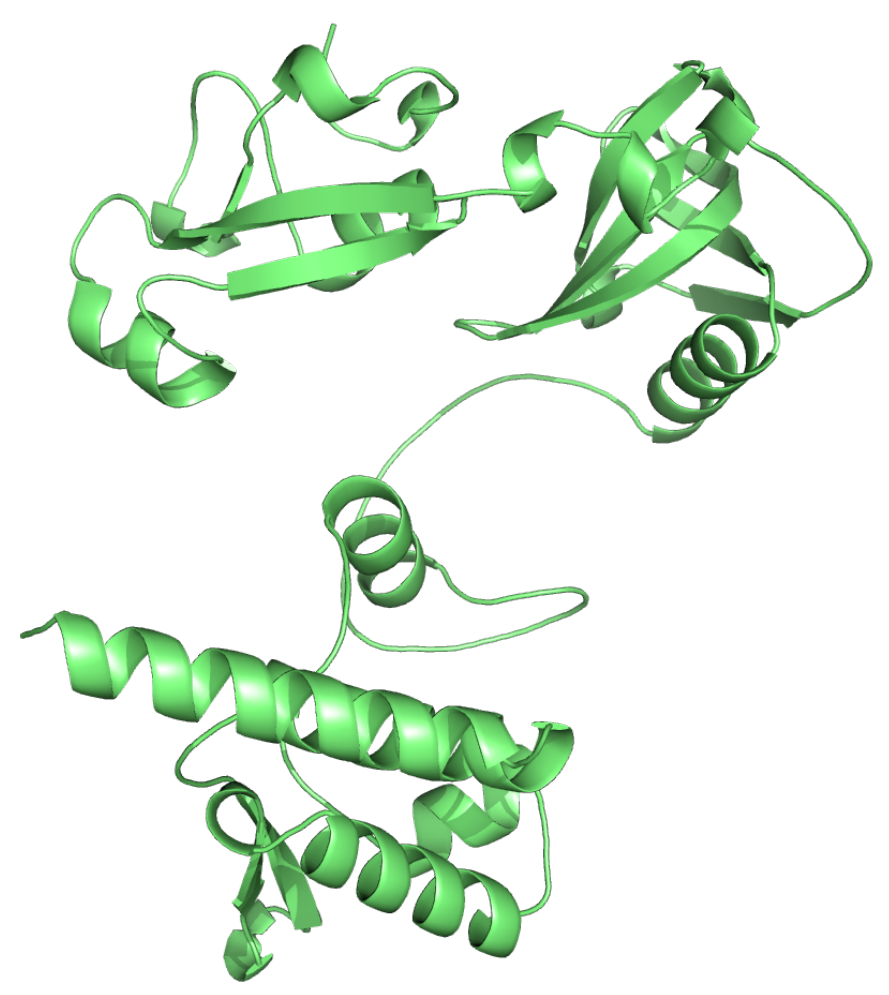

\section{VchIND5 model (AlphaFold)}

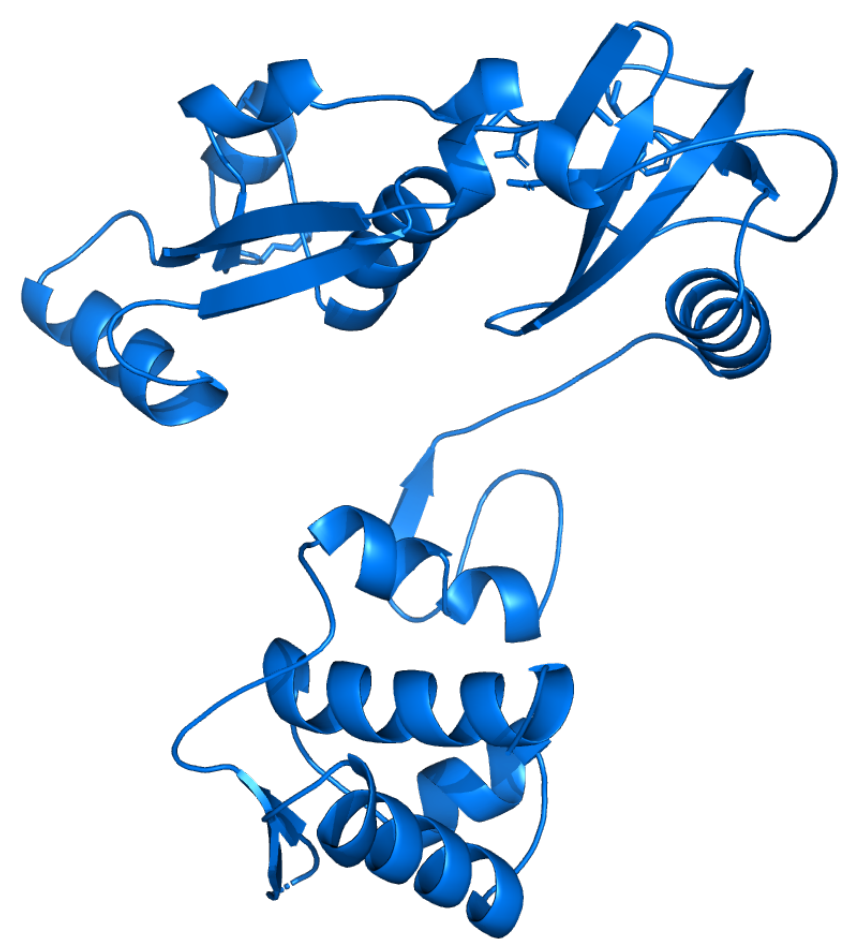

BrxR structure 


\section{PDB ID}

\section{Data Collection}

Space group

Unit cell
$a, b, c$
alpha, beta, gamma
Wavelength $(\AA)$
Resolution range $(\AA)$
Unique reflections
R-merge
I/sigma(I)
Multiplicity
Completeness (\%)
Mean $B$ value

R-meas

R-pim

CC1/2

Chi^2

\section{Refinement}

Resolution (Å)

Completeness for resolution used (\%)

R-work

R-free

Number of non-hydrogen atoms

macromolecules

ligands

solvent

Protein residues

RMS(bonds)

RMS(angles)

Ramachandran favored (\%)

Ramachandran allowed (\%)

Ramachandran outliers (\%)

Clashscore

Average B-factor

macromolecules

ligands

solvent

\section{BrxR (unbound)}

7T8L

P 1211

$65.3,48.67,96.11$

$90,102.28,90$

0.9795

93.7-2.08 (2.12-2.08)

35573

$0.066(0.239)$

$0.091(0.319)$

0.051 (0.176)

$0.996(0.862)$

$12.27(1.3)$

$1.114(0.779)$

$3.0(3.2)$

99.7 (99.8)

30.902

\section{BrxR (DNA bound)} 7T8K

\author{
C 121
}

151.6, 71.5, 87.4

$90,105.1,90$

0.9763

64.2-2.3 (2.38-2.3)

38731

$0.075(0.572)$

0.105 (0.668)

$0.042(0.264)$

$0.975(0.847)$

$11.3(2.6)$

$1.375(0.655)$

$6.2(6.1)$

97.5 (100.0)

48.65

64-2.3

63.92-2.0

99.45

0.1910

95.25

0.1958

0.2376

4564

5588

5445

4250

156

158

26

117

563

0.009

1.571

0.004

0.73

97.5

2.58

0.4

4.83

34.65

33.42

35.35

35.19
2.5

0

12.84

55.04

55.04

68.08

51.91 


\section{Supplementary Table S1. Primers for Molecular Biology and Subcloning}

\begin{tabular}{|c|c|}
\hline Primer Name & Oligonucleotide sequence $\left(5^{\prime}->3^{\prime}\right)$ \\
\hline Ac_BREX block1 $\mathrm{R}$ & 5'-TCTAGATCTTCCCCGGGGATCCTTAAT CCACCCCTAACCCTTTAAACACC-3' \\
\hline Ac_BREX block1 F & 5'-ACTAGACCTTGATGAGGTCGTTGCATG-3' \\
\hline Ac BREX block2 $\mathrm{R}$ & 5'-CATGCAACGACCTCATCAAGGTCTAGT-3' \\
\hline Ac_BREX block2 F & 5'-GACCAGCGACTGAGAAAGAGCTGTTTG-3' \\
\hline Ac_BREX block3 R & 5'-CAAACAGCTCTTTCTCAGTCGCTGGTC-3' \\
\hline Ac_BREX block3 $\mathrm{F}$ & 5'-TGCCTGCAGTTAAGGTTTAACATATGA CAGCAGACAAGCATGAAGTG-3' \\
\hline BrxA del $R$ & 5'-ATTGCTGCCCCTCTACAAGGCCAATCT CCTATGAGGCCT-3' \\
\hline BrxA del $F$ & 5'-TTGTAGAGGGGCAGCAATGAG-3' \\
\hline BrxB del $R$ & 5'-TATGGCTATTTGTCGTGCTGCTGCCCC TCTACAACTAAC-3' \\
\hline BrxB del F & 5'-GCACGACAAATAGCCATAAAAATTCGG-3' \\
\hline BrxC del $\mathbf{R}$ & 5'-CGATATCCCTAACCCAAGCTCAATCAA TTCCCCGAATTTTTATGGCTA-3' \\
\hline BrxC del F & 5'-CTTGGGTTAGGGATATCGGCATC-3' \\
\hline PglX del $\mathbf{R}$ & 5'-GTACCGGAGAAAAAACATTCATTAAAAACCTTCAATTGATTGCTGATG-3' \\
\hline PgIX del F & 5'-TGAATGTTTTTTCTCCGGTACTAG-3' \\
\hline PglZ del R & 5'-CGCTCTATCGTTAATTCGTTAGCAACG ACCTCATCAAGGTCT-3' \\
\hline PglZ del F & 5'-TAACGAATTAACGATAGAGCGTAGCCTTAC-3' \\
\hline BrxL del R & 5'-CTTCCCCGGGGATCCGTAGGCTACGCTCTATCGTTACGTTAT-3' \\
\hline BrxL del F & 5'-GGATCCCCGGGGAAGATCTAG-3' \\
\hline BrxR del $\mathbf{R}$ & 5'-TCTAACATGGCCAATCTCATGTTAAAC CTTAACTGCAGGCATGC-3' \\
\hline BrxR del $F$ & 5'-GAGATTGGCCATGTTAGAACAATTTCATTA-3' \\
\hline brxRBD_for1 & 5'-GGGTAGTCTTGATCACATTGTTTATTGACCGATTGTTCTATGACAGCAGACAAGCATGAA-3' \\
\hline brxRBD_delR_for1 & 5'-TGATCACATTGTTTATTGACCGATTGTTCTATGTTAGAACAATTTCATTATGACAGTGAC-3' \\
\hline brxRBD_rev1 & 5'-TACGGTATTGTGAGGGCAGGTTAAACATATC CATGAGAATTACAACTTATATCGTATGGG-3' \\
\hline brxRBD_for2 & 5'-CCGTAAAAATAATTTACTGTATATTCATTGTAGGGTAGTCTTGATCACATTGTTTATTG-3' \\
\hline brxRBD_rev2 & 5'-TACAATGAATATACAGTAAATTATTTTTACGGTATTGTGAGGGCAGG-3' \\
\hline brxRBD_delR_for2 & 5'-ITTACTGTATATTCATTGTAGGGTAGTCTTGATCACATTGTTTATTGACCGATTG-3' \\
\hline brxRBD_delR_rev2 & 5'-ACCCTACAATGAATATACAGTAAATTATTTTTACGGTATTGTGAGGGCAG-3' \\
\hline
\end{tabular}


bioRxiv preprint doi: https://doi.org/10.1101/2021.12.19.473356; this version posted December 19, 2021. The copyright holder for this preprint (which was not certified by peer review) is the author/funder. All rights reserved. No reuse allowed without permission.

\section{Supplementary Table S1. Primers for Molecular Biology and Subcloning}

Supplementary Table 1. Oligonucleotide sequence of PCR primers used in this study. Sequence in bold indicate nucleotides inserted upstream of BREX operon in PACYC plasmid constructs. Underlined nucleotides indicate overlapping sequence used for HiFi assembly. 